\title{
La protection des lanceurs d'alerte au Canada et au Québec : un état des lieux
}

\author{
Jeanne Simard ${ }^{a}$, France Desjardins $^{\mathrm{b}}$, Marc-André Morency $^{\mathrm{c}}$
}

"If you see something, say something " should not just be reserved for public security, but should apply to all cases of secrecy where the public interest is at stake $e^{1}$. - Stiglitz et Pieth, 2016, p. 19

RÉSUMÉ. Le présent article a pour objectif d'examiner comment le droit encadre le processus de divulgation et protège au Canada et au Québec les lanceurs d'alerte contre les mesures de représailles dans les organisations des secteurs privé et public. La première partie, au contenu plus conceptuel, tente de distinguer les types de comportements d'alerte, leur légitimité et leur utilité sur les plans social et organisationnel. La deuxième partie, au contenu cette fois plus juridique, brosse un tableau des différents cadres légaux qui ont été adoptés au fil des ans au Canada et au Québec pour encourager et protéger les lanceurs d'alerte. Ces cadres sont souvent flous et diffèrent selon que la divulgation est de compétence fédérale ou provinciale, s'appliquent au secteur public ou privé et selon le type de renseignements divulgués. En matière d'alerte, on doit retenir que, même si la protection des lanceurs d'alerte a été enrichie à plusieurs égards au fil des ans, cette protection pourrait encore être améliorée, car elle présente certaines lacunes et l'effectivité du droit dans ce domaine est souvent mise à mal.

\begin{abstract}
The purpose of this article is to examine how the law governs the disclosure process and protects whistleblowers in Canada and Quebec against reprisal measures in private and public sector organizations. The first part, with a more conceptual content, attempts to distinguish the types of alert behaviour, their legitimacy and their usefulness socially and organization wise. The second part, that is more legal in content, paints a picture of the different legal frameworks that have been adopted over the years in Canada and Quebec to encourage and protect whistleblowers. These frameworks are often vague and differ depending on whether the disclosure is under federal or provincial jurisdiction, whether it applies to the public or private sector, and depending on the type of information disclosed. In terms of whistleblowing, it should be remembered that, even if the protection of whistleblowers has been increased in several respects over the years, this protection could still be improved, because there are certain weaknesses and the effectiveness of the law in this area is often undermined.
\end{abstract}

\section{Introduction}

Au cours des dernières décennies, de nombreux cas de corruption, d'évasion fiscale, d'atteinte à la vie privée et de dommage à l'environnement ou à la santé publique ont démontré que les mécanismes de gouvernance et de surveillance des organisations publiques et privées se trouvent souvent défaillants dans la prévention et la sanction des comportements déviants ou dommageables. Il est parfois difficile de prévenir ou trop tard pour intervenir, car l'asymétrie informationnelle entre les acteurs, aussi bien économiques que politiques, demeure tout à fait considérable.

Devant cette faiblesse inhérente à nos modalités de gouvernance, on s'est intéressé un peu partout dans le monde, depuis la fin des années 1990 (mais beaucoup plus tôt aux États-Unis), à des mécanismes supplémentaires de diffusion de l'information pertinente permettant d'identifier et de faire cesser le plus rapidement des actes répréhensibles ou déviants. On

\footnotetext{
${ }^{a}$ Professeure, membre du Laboratoire de recherche et d'intervention en gouvernance des organisations (LARIGO), Université du Québec à Chicoutimi

${ }^{\mathrm{b}}$ Chargée de cours, membre du Laboratoire de recherche et d'intervention en gouvernance des organisations (LARIGO), membre associé du Laboratoire d'études multidisciplinaires en gestion de projet (LemGP), Université du Québec à Chicoutimi

c Sociologue, décédé en 2019, professeur au département des sciences humaines et sociales de 1978 à 2004, Université du Québec à Chicoutimi
} 
fait ainsi appel à ceux qui disposent d'information pertinente, souvent de première main. Ces personnes peuvent être des employés œuvrant à l'intérieur des organisations privées ou publiques, mais aussi des personnes extérieures (outsiders) des organisations (des victimes et/ou des témoins) ou encore des organisations vouées à la défense de «droits» collectifs divers.

Ces diffuseurs d'information, qu'on appelle souvent indistinctement «dénonciateurs», «divulgateurs», «lanceurs d'alerte» ou «whistleblowers» ${ }^{2}$, ont défrayé les manchettes, alimenté les libraires ou réveillé la conscience collective en relatant des faits ou actes répréhensibles portant atteinte à la communauté3. Or, cet historique a abondamment révélé les lacunes, l'inégalité, voire la sévérité des réactions organisationnelles et juridiques à leur endroit (Fines, 2013; Hartmann, 2014). Plusieurs ont fait l'objet d'ostracisme, ont été rétrogradés, congédiés ou ont dû faire face à des accusations criminelles, ce qui leur a fait subir de lourdes séquelles, malgré leur contribution au bien public.

Pour donner quelques exemples, au Québec, la Commission d'enquête sur l'octroi et la gestion des contrats publics dans l'industrie de la construction a permis d'entendre de nombreux témoignages portant sur les pratiques de collusion et de corruption dans l'industrie québécoise de la construction œuvrant dans le champ municipal. Le témoignage d'une ingénieure de la firme Genivar, punie pour avoir divulgué la corruption, lui a valu d'être félicitée par la juge Charbonneau, présidente de cette commission. Plus récemment, le congédiement du lanceur d'alerte Louis Robert, agronome au ministère de l'Agriculture, des Pêcheries et de l'Alimentation du Québec (MAPAQ), a soulevé l'indignation de la population générale et de certains politiciens. Son message visait l'ingérence des entreprises privées dans la recherche publique sur l'usage des pesticides en agriculture. Il a pu réintégrer son ancien poste quelques mois plus tard, mais non sans avoir subi de graves préjudices. En avril 2021, une employée d'un centre d'hébergement de longue durée (CHSLD) a été congédiée pour avoir envoyé des photos de résidents à un journaliste pour dénoncer leurs conditions de vie durant la pandémie de COVID-19. Pour l'employeur, cette dernière n'a pas respecté les informations confidentielles des personnes résidentes au CHSLD parce qu'elle a en quelque sorte dévoilé leur adresse. Quelques jours plus tard, la ministre responsable des Aînés et des Proches aidants refusait de prendre la défense de l'employée en affirmant qu'elle n'avait pas suivi les procédures internes établies pour dénoncer certaines situations. Les jours suivants, le premier ministre du Québec a mandaté son ministre de la Santé et des Services sociaux de faire la lumière sur cette situation en faisant référence au cas du lanceur d'alerte Louis Robert. De plus, tous les partis siégeant à l'Assemblée nationale ont adopté une motion afin de souligner l'importance de prendre les mesures nécessaires pour protéger les professionnels de la santé des risques de représailles auxquels ils s'exposent en dénonçant des situations inacceptables.

La protection du lanceur d'alerte par la justice s'avère donc une question des plus délicates. Elle exige, à de nombreuses occasions, de trouver un équilibre entre des valeurs, des droits et des obligations souvent contradictoires, soit entre la liberté d'expression du lanceur d'alerte et ses obligations de loyauté ou de réserve envers son employeur, si ce dénonciateur est un salarié. L'examen de cette vaste question impose aussi de marquer les particularités des différents contextes éthiques et politiques où la divulgation se produit. Car si l'on peut, en conscience, décider de bonne foi de divulguer de l'information au sujet de pratiques douteuses portant atteinte à la démocratie, à la justice ou à l'environnement, à l'autre extrémité du spectre, certains pourraient vouloir «divulguer» de l'information dans un but d'enrichissement personnel ou encore pour nuire à autrui, à son employeur, à un concurrent, à l'économie ou à la société en général. Bref, les législateurs à travers le monde doivent faire des choix politiques et sociaux importants lorsque vient le temps d'adopter un cadre normatif qui encourage et protège la divulgation. Il dépendra souvent du contexte social et historique de leur pays.

Le présent article a pour principal objectif d'examiner comment le droit canadien et québécois encadre le processus de divulgation, et protège les lanceurs d'alerte contre les mesures de représailles dans les organisations des secteurs privé et public au Canada et au Québec. Pour faire cette démonstration, l'article sera divisé en deux grandes parties.

La première partie, au contenu plus conceptuel, mobilise le regard sociologique et juridique, avec pour objet de distinguer les types de comportements d'alerte, leur légitimité ainsi que leur utilité sur les plans social et organisationnel. Cette partie 
comprendra trois sous-sections. Dans la première sous-section, nous verrons les trois grandes questions de base que devraient se poser les législateurs lorsqu'ils décident d'adopter un cadre normatif pour encourager et protéger les lanceurs d'alerte. Leurs choix auront des répercussions importantes sur l'étendue et la portée de leur loi. Dans la deuxième sous-section, nous identifierons des formes de contestation apparentées à la divulgation, par exemple la fuite d'information (leakage), le journalisme d'enquête et la délation. Dans la troisième sous-section, nous verrons que, peu importe l'objet de la divulgation, un équilibre doit, dans certaines situations, être trouvé entre plusieurs valeurs, dont la liberté d'expression et le devoir de loyauté envers un employeur ou encore vis-à-vis des parties prenantes du corps social ambiant.

Dans la deuxième partie de l'article, nous verrons que les cadres légaux qui ont été adoptés au fil des ans pour encourager et protéger les lanceurs d'alerte sont souvent flous et différents selon que la divulgation est de compétence fédérale ou provinciale, dans le secteur public ou privé et selon le type de renseignements divulgués.

Bref, il devient de plus en plus pressant que le Canada et le Québec adoptent un corpus normatif clair et des pratiques uniformes dans leur champ de compétence respectif. Dans la situation actuelle, il n'est pas surprenant que certaines personnes détenant des informations importantes préfèrent garder le silence (Martin-Bariteau et Newman, 2018). Pourtant, les programmes de divulgation dans les organisations publiques et privées constituent des outils de gestion non négligeables dans la lutte contre la corruption ainsi que dans la protection du public et de l'environnement.

\section{La divulgation et le lanceur d'alerte : une indétermination de sens}

Lorsque nous consultons la littérature scientifique, les textes de loi, les journaux ou encore les documents de régie interne des organisations à travers le monde, nous rencontrons un spectre de définitions qui donnent une portée plus ou moins large à la notion de divulgation ainsi qu'aux critères permettant d'identifier un lanceur d'alerte ${ }^{4}$. En effet, le phénomène de prise de parole ou d'alerte, encore en pleine construction, se nourrit d'une large variété d'expériences individuelles et collectives. On est en présence d'un univers conceptuel complexe qui évoque des rapports de force ainsi que des conflits de loyauté, voire de trahison aux formes multiples (Schehr, 2008). De nombreuses « définitions de situation » provenant des traditions morales, éthiques, politiques, légales et économiques s'affrontent et cherchent à s'en approprier le destin.

De plus, en raison de la culture et de l'histoire particulières des pays et des communautés, mais aussi des différents régimes juridiques et institutions soutenant l'État de droit, on n'observe pas encore de cadre conceptuel commun sur le plan international pour définir ce phénomène de prise de parole, pour en fixer le rôle et la portée, ni pour déterminer le statut et les protections juridiques du lanceur d'alerte, et ce, en dépit des pressions d'un réseau mondial d'organisations non gouvernementales (ONG) de premier plan travaillant à les soutenir et à les protéger ${ }^{5}$.

Pour notre part, la définition de la divulgation (ou dénonciation) suggérée par les auteurs Culiberg et Mihelic (2017) nous apparait comme le meilleur cadre conceptuel pour explorer et comprendre ce phénomène de prise de parole. Elle offre la flexibilité nécessaire pour contenir plusieurs cas de figure de la divulgation, tout en la limitant aux situations qui impliquent de possibles actes répréhensibles au sein d'une entreprise ou d'une organisation publique ou privée : $^{2}$

La dénonciation est une divulgation faite par une personne ayant un accès privilégié aux données d'une organisation ou à des informations sur un acte réprébensible, qui implique l'organisation [...], à des personnes ou des organisations qui peuvent être en mesure d'effectuer une action». (Culiberg et Mihelic, 2017, p. 788, faisant référence à Jubb, 1999 et à Near et Miceli, 1995, trad. libre, italique ajouté)

Afin de bien comprendre la portée et l'efficacité des lois adoptées au Canada et au Québec pour encourager les processus de divulgation ainsi que pour accompagner et protéger les lanceurs d'alerte pendant et après la divulgation, il est important d'aborder cette problématique de manière dynamique en examinant trois grandes questions de base qui se posent en analysant attentivement la définition proposée par Culiberg et Mihelic : Qui 
peut divulguer? Quels actes répréhensibles peuvent faire l'objet d'une divulgation? Qui peut recevoir une divulgation? (Brown, 2013; Clark, 2012; Culiberg et Mihelic, 2017).

Ces questions nous permettent de conserver le recul épistémologique nécessaire pour ne pas ramener indûment la fonction sociale de la divulgation à l'horizon des textes juridiques nationaux visant à protéger les personnes qui décident de s'y engager (Lavite, 2016), le phénomène étant beaucoup trop complexe. Cette solution, commode pour certains, «serait évidemment inadaptée et même inopportune - en tout cas prématurée - dans un contexte où l'on cherche précisément à élargir le champ des personnes protégées par les textes » (Lochak, 2016, paragr. 5), mais aussi à élargir le spectre des actes répréhensibles pouvant faire l'objet d'une divulgation ainsi que les espaces disponibles pour le faire.

\subsection{Trois questions pour délimiter les contours du phénomène de la divulgation}

\subsubsection{Qui peut divulguer?}

Au cours des dernières décennies, la définition de «divulgateurs» (ou lanceurs d'alerte) de Near et Miceli (1995) a été abondamment citée par la littérature, soit :

des membres d'une organisation actuelle ou ancienne, ou des personnes dont les activités ou les actions sont sous l'autorité de l'organisation, et lesquels n'ont pas de pouvoir pour prévenir ou faire cesser les actes répréhensibles de l'organisation, qu'ils choisissent ou non de demeurer anonymes dans la dénonciation et qu'ils occupent ou non des fonctions organisationnelles qui ordonnent officiellement la dénonciation. (Near et Miceli, 1995, p. 2, trad. libre)

À l'intérieur des paramètres de cette définition traditionnelle du lanceur d'alerte, on retrouve, il va sans dire, la plupart des employés d'une organisation (publique ou privée), et ce, peu importe leur rang hiérarchique. À titre d'exemples, ces employés peuvent être gestionnaires, professionnels, cadres ou vérificateurs (Lewis, 2011). Selon certains auteurs (Jubb, 1999; Miceli et collab., 2014), cette définition se justifie par le fait que seuls les initiés (c.-à-d. les membres d'une organisation) ont la crédibilité nécessaire pour effectuer la divulgation d'un acte répréhensible affectant la bonne gouvernance de leur organisation et, par conséquent, doivent faire l'objet d'une protection. Considérant le contexte des organisations aujourd'hui, cette définition peut également inclure les salariés ou travailleurs atypiques, soit des personnes sous contrats ponctuels (temporaires ou à temps partiel) pour accomplir des mandats précis, mais également les stagiaires, les bénévoles ainsi que les anciens et futurs employés (Lachapelle, 2019, p. 226) 7.

Or, de plus en plus de voix s'élèvent pour que cette définition traditionnelle (axée strictement sur la relation de travail) soit élargie et pour que l'on considère désormais comme lanceurs d'alerte les personnes qui ne sont pas nécessairement liées à une organisation par un contrat d'emploi, soit : les consommateurs, les clients, les fournisseurs, les distributeurs, les investisseurs, les consultants ou tout autre groupe pertinent (Smaili et Arroyo, 2019; Culiberg et Mihelic, 2017; Ayers et Kaplan, 2005)8 .

Ces lanceurs d'alerte extérieurs (outsiders) prennent de plus en plus d'importance dans le combat des faits répréhensibles au sein des organisations (Miceli et collab., 2014). Ils apportent un autre point de vue et un éclairage factuel qui complètent souvent celui des initiés ${ }^{9}$. Ces personnes, en dénonçant, peuvent aussi « se trouver, eu égard à leur relation professionnelle, dans une situation de vulnérabilité économique» (Lachapelle, 2019, p. 226). Ainsi, la figure du lanceur d'alerte peut même être élargie à tout citoyen concerné (De Maria, 1995), à tout acteur social qui met en lumière un acte répréhensible lié à une organisation (Dyck et collab., 2007; Culiberg et Mihelic, 2017; Smaili et Arroyo, 2019).

\subsubsection{Quels sont les actes répréhensibles qui peuvent être divulgués?}

Quels types d'actes répréhensibles peuvent être divulgués? Quelles informations sur ces actes présentent un intérêt public? Le spectre des comportements répréhensibles peut être très large et, là aussi, modifier considérablement la définition et la portée de la divulgation.

Certains ont une acception restrictive des actes répréhensibles pouvant être divulgués, couvrant 
des faits bien ciblés, par exemple uniquement les actes criminels graves (corruption, fraude, collusion, vol, détournement de biens publics) et les infractions pénales (environnement, santé et sécurité au travail $)^{10}$. Cette acception est nettement privilégiée dans les législations du monde anglosaxon, qu'on appelle communément whistleblowing.

Par contre, d'autres acceptions couvrent un champ d'application matériel plus général, pouvant aller de violations d'une loi (pas nécessairement criminelle ou pénale), de menaces pour l'intérêt public et de risques réels pour la société et son environnement aux simples problèmes de comportement (harcèlement, discrimination, abus de pouvoir) et d'interaction entre personnes (Comité permanent des opérations gouvernementales et des prévisions budgétaires, 2017, citant le commissaire à l'intégrité du secteur public du Canada, Joe Friday).

Dans une acception encore plus englobante, la divulgation (communément appelée en France «l'alerte éthique») devient en quelque sorte un acte de vigilance active, un outil d'intervention pour tous les citoyens qui va au-delà de la simple dénonciation d'un acte illégal, immoral ou illégitime à l'intérieur d'une organisation. L'alerte éthique ouvre alors un processus de discussion critique et de mobilisation qui amène des personnes ou des groupes à dévoiler à la conscience collective, aux pouvoirs publics ou aux autorités des pratiques contraires au bien commun, à l'intérêt général. Cet intérêt général peut être défini de plusieurs manières, car il est «à la fois un concept juridique et une valeur» (Piraux, 2008, paragr. 173). Selon certains, l'alerte éthique devrait comprendre toute démarche de précaution ou de vigilance démocratique de tout citoyen responsable : «Toute personne, groupe ou institution qui, percevant les signes précurseurs d'un danger ou d'un risque, interpelle une ou plusieurs puissances d'action, dans le but d'éviter un enchaînement catastrophique, avant qu'il ne soit trop tard» (Chateauraynaud, 2013, s. p.).

\subsubsection{Quelle personne ou entité peut recevoir une divulgation?}

Un aspect important de la procédure de divulgation est de préciser quelle personne ou institution est admissible à la recevoir (Andrade, 2015). D'emblée, on doit distinguer trois formes (trois niveaux, diront certains) de divulgation : 1) celle qui est faite à l'intérieur de l'organisation, 2) celle qui s'adresse à l'extérieur de l'organisation et 3) celle qui est faite directement au public, souvent par l'entremise des médias (Lachapelle, 2019).

\section{Divulgation faite à l'intérieur de l'organisation}

Dans ce cas, la divulgation est transmise à une personne (un supérieur, un directeur, un responsable de l'éthique, un cadre) ou à une entité (comité d'éthique, comité d'audit, contrôle de service interne) au sein de l'organisation qui est désignée à cette fin par l'employeur. La divulgation interne peut même se faire par le biais d'une ligne téléphonique confidentielle mise à la disposition des employés. L'organisation peut décider d'une procédure formelle de divulgation interne, qui comprend une façon de faire le suivi de la divulgation ainsi que d'appliquer les mesures de confidentialité et les modalités de protection du lanceur d'alerte, ou encore se voir imposer une telle procédure. Or, "[la] dénonciation faite à l'intérieur même de l'entreprise est un mécanisme connu de longue date et fait souvent partie des programmes de conformité» (Tchotourian, 2016, p. 1). L'organisation qui dispose d'un processus de divulgation interne clair et connu de tous peut rapidement rectifier des actions répréhensibles avant qu'elles ne soient connues du public (Culiberg et Mihelic, 2017).

\section{Divulgation qui s'adresse à l'extérieur de l'organisation}

Dans ce deuxième cas, la divulgation dite externe est faite à une personne ou à une instance qui n'est pas réputée faire partie de l'organisation. Le destinataire de la divulgation doit avoir «la capacité réelle de mettre fin à cette situation » (Jubb, 1999, trad. de Foegle, 2016, paragr. 16), comme cela est le cas pour certaines autorités paragouvernementales au Québec telles que l'Autorité des marchés publics, le Protecteur du citoyen, le Commissaire à la lutte contre la corruption, l'Unité permanente anticorruption ou encore la police. Ces instances externes sont souvent désignées comme telles dans les différentes lois protégeant les lanceurs d'alerte.

Divulgation faite directement au public, souvent par l'entremise des médias

Enfin, dans le troisième cas, la divulgation « peut se faire [...] auprès d'un journaliste, d'une organisation non gouvernementale, d'un parlementaire ou directement via une page web ou une plateforme 
en ligne» (Lachapelle, 2019, p. 231). La société est ainsi informée de l'acte répréhensible reproché à l'organisation. Or, comme nous allons le voir un peu plus loin dans ce texte, tant sur le plan éthique que juridique, la divulgation publique d'actes répréhensibles est souvent considérée comme déloyale, voire moralement ambiguë (Laframboise, 1991; Tait, 1997).

De fait, cette distinction se trouve marquée dans les législations qui en traitent, protégeant les uns, négligeant souvent les autres. Pour cette raison, les tribunaux et plusieurs lois privilégient désormais une approche par paliers, soit la divulgation traitée au départ par des mécanismes internes de l'organisation, puis par des instances externes, s'il y a lieu de croire que la question ne peut être divulguée de manière confidentielle au sein de son secteur. En dernier recours, la divulgation est publique, si des conditions strictes sont remplies.

\subsection{Diverses formes de contestation apparentées à la divulgation}

D'autres formes de participation et de contestation citoyennes peuvent s'avérer proches parentes de la divulgation. Il faut toutefois considérer que, si la fuite d'information (leakage), le journalisme d'enquête ou la délation en partagent certaines caractéristiques, ils ne peuvent y être pleinement assimilés et devraient faire - ou font - l'objet d'une protection juridique particulière :

Il y a incontestablement un effet de mode dans cette tendance à labelliser comme «alerte éthique» des formes de rébellion ou de contestation qui peuvent être très différentes les unes des autres - un effet de mode qui va de pair avec la valorisation de la figure du « rebelle» : car le lanceur d'alerte peut être vu comme celui qui se rebelle contre les ordres reçus, contre sa hiérarchie, contre la conjuration du silence. (Lochak, 2016, p. 2)

\subsubsection{Fuite d'information}

La fuite d'information ou de données (leakage) nécessite d'enfreindre délibérément les lois relatives au secret gouvernemental ou militaire, et consiste «à rendre publiques des informations classifiées », et ce, au nom d'une valeur suprême et très spécifique : la transparence à tout prix de l'action publique
(Lochak, 2016). C'est pour cette raison que la notion de leakage se trouve le plus souvent apposée aux fuites provenant d'agents de services de renseignement, de membres de la diplomatie et des armées. Le fuiteur (leaker) revendique pour les citoyens le droit d'accéder aux documents et aux procédures de leur gouvernement afin de pouvoir les comprendre, les évaluer et les juger ${ }^{11}$.

On peut penser que cette pratique peut avoir inspiré les actions de divulgation de WikiLeaks, une ONG fondée par Julian Assange, ou encore du collectif Anonymous. Contrairement au lanceur d'alerte, le fuiteur d'information en fait une "profession» (Foegle, 2016). Selon certains auteurs, ce n'est pas la désobéissance aux lois relatives au secret qui doit distinguer le fuiteur du dénonciateur, mais plutôt les fins générales poursuivies par ce dernier (Foegle, 2016; Lochak, 2016).

\subsubsection{Journalisme d'enquête}

Le journaliste professionnel et accrédité comme tel est une "personne dont l'occupation principale consiste à contribuer directement et moyennant rétribution, soit régulièrement ou occasionnellement, à la collecte, la rédaction ou la production d'informations en vue de leur diffusion par les médias, ou tout collaborateur de cette personne» (art. 2 de la Loi sur la protection des sources journalistiques, LC 2017, chap. 22). Son rôle consiste à communiquer des informations au public. Le journaliste se trouve parfois alimenté par ces fuites et contestations, pratique la recherche soutenue et la divulgation de l'information et, in fine, l'étalement à grande échelle de comportements scandaleux en matière économique et politique.

Le journaliste «n'est pas lui-même, stricto sensu, un lanceur d'alerte, puisque la diffusion de l'information fait partie de son métier» (Lochak, 2016, p. 4). Il est un professionnel de la transparence. Par exemple, en 2016, la diffusion des Panama Papers représentait le fruit d'une collaboration entre professionnels regroupés dans le Consortium international des journalistes d'investigation. Les Panama Papers traitaient de millions de documents confidentiels produits par une firme d'avocats panaméenne. Le fruit de ce travail d'enquête: le dévoilement d'informations sur plus de 214000 sociétés extraterritoriales (offshore) ainsi que les noms 
des actionnaires de ces sociétés profitant de fait d'une vaste zone de non-droit ${ }^{12}$.

\subsubsection{Délation}

La dernière forme de contestation apparentée à la divulgation est la délation, qui traduit des motivations essentiellement égocentriques du délateur :

Il y a délation lorsqu'on dénonce quelqu'un par intérêt égoïste (par exemple pour toucher une prime, de la part des services fiscaux) ou, plus généralement, pour quelque autre raison que l'exigence de justice ou la volonté de protéger les victimes (par exemple par haine ou par jalousie). C'est en quoi la délation, même légalement autorisée ou encouragée [...], est toujours moralement condamnable. Elle peut parfois servir la justice. Elle n'en est pas moins méprisable. (Comte-Sponville, 2017, s. p.)

Même si elle peut être socialement utile et efficace dans certaines situations, la délation n'a pas bonne réputation dans les communautés et les pays ayant vécu la guerre, l'occupation ou la dictature. Un mouchard, un stooler ou un rapporteur sont des termes péjoratifs bien connus dans les écoles, les prisons, etc. Cette connotation négative peut provenir du fait que la personne qui fait la dénonciation n'est pas mise en cause.

Le droit criminel et le droit pénal canadiens permettent qu'un accusé devenant un témoin délateur (ou un témoin repenti) dénonce ses anciens complices pour échapper à la prison ou encore pour bénéficier d'une réduction de peine, d'une compensation financière ou d'une protection particulière (changement d'identité) :

Sur les plans légal et moral, il va de soi que le témoin délateur n'est pas un citoyen guidé par sa conscience sociale et hanté par le remords ou le repentir. Il collabore seulement dans la mesure où il en tire des avantages (Boisvert, 2005 , p. 13, citant le Groupe de travail sur l'administration de la justice en matière criminelle, 1991, p. 69) ${ }^{13}$.

\subsection{Les loyautés en conflit pour le salarié lanceur d'alerte}

Peu importe l'objet de la divulgation, un équilibre doit être trouvé entre plusieurs loyautés, entre plusieurs valeurs. C'est pourquoi le lanceur d'alerte salarié d'une organisation se trouve généralement dans une situation où la décision éthique qu'il doit prendre de divulguer ou non un acte répréhensible attribuable à son employeur peut s'avérer extrêmement difficile à réaliser. Il doit prendre des risques pour lui, pour son entourage et pour ses proches.

Dans la plupart des pays, un salarié, en plus d'être tenu d'exécuter son travail avec prudence et diligence, doit agir avec loyauté vis-à-vis de son employeur, même après la cessation de son emploi (art. 2088 du Code civil du Québec). Ce devoir de loyauté résulte du principe selon lequel la bonne foi et la confiance doivent gouverner l'ensemble des relations contractuelles. "L'obligation de loyauté ainsi que le principe de bonne foi contraignent ainsi le salarié à retenir ses propos afin d'éviter d'attaquer la réputation de son employeur ou de lui nuire» (Rancourt et Cloutier, 2008, s. p.). Ils sont inscrits dans les lois ou dans les contrats individuels et collectifs de travail (Brunelle et Samson, 2005) :

Partant du postulat que l'employeur, à savoir l'organisation de manière générale, est dans une position qui lui permet d'avoir la vision la plus complète de la situation, l'employé loyal doit projeter l'image d'un individu qui sait ce qu'il doit à celui qui l'emploie et qui lui fait confiance, même au-delà de ce qu'il voit. (Cailleba, 2019, p. 248)

Pour les employées du secteur public québécois, la Loi sur la fonction publique (chap. F-3.1.1, art. 126) encadre aussi leur travail. Cette loi établit les devoirs et les obligations des fonctionnaires, dont le devoir de discrétion et celui de réserve comme suit:

Art. 6. Sous réserve des dispositions relatives à l'accès à l'information et à la protection des renseignements personnels, le fonctionnaire est tenu à la discrétion sur ce dont il a connaissance dans l'exercice de ses fonctions.

Art. 11. Le fonctionnaire doit faire preuve de réserve dans la manifestation publique de ses opinions politiques.

De plus, le Règlement sur l'ethique et la discipline dans la fonction publique (chap. F-3.1.1, art. 126) précise les obligations du fonctionnaire en matière de manifestations publiques : 
Art. 8. Le fonctionnaire qui se propose de publier un texte ou de se prêter à une interview sur des questions portant sur des sujets reliés à l'exercice de ses fonctions ou sur les activités du ministère ou de l'organisme où il exerce ses fonctions doit préalablement obtenir l'autorisation du sous-ministre ou du dirigeant de l'organisme.

Il y a aussi une pléthore de lois qui protègent « une opacité estimée légitime» comme les secrets d'État, les secrets commerciaux, les secrets professionnels, les renseignements personnels, etc. (De Quenaudon, 2015, p. 295). Si un employé contrevient à son devoir de loyauté ou de confidentialité, il s'expose à des mesures disciplinaires importantes.

D'autres lois comme la Charte canadienne des droits et libertés et la Charte québécoise des droits et libertés de la personne (RLRQ, chap. C-12) ou encore la Déclaration universelle des droits de l'homme consacrent le droit constitutionnalisé à la liberté d'expression. Cette liberté contribue à protéger et à développer «une démocratie dynamique qui accepte et encourage la participation de tous » (R. c. Keegstra, [1990] 3 RCS 697, p. 766).

Le droit de lancer une alerte constitue une manifestation de cette liberté d'expression et est souvent reconnu à cet effet dans le préambule des différentes lois adoptées au fédéral ou au Québec pour protéger les lanceurs d'alerte contre les mesures de représailles des employeurs. Dès 2005, la Cour suprême du Canada, dans l'affaire Merk. c. Association internationale des travailleurs en ponts, en fer structural, ornemental et d'armature, section locale 771 , a reconnu l'importance de ces lois :

[14] Les lois sur la protection des dénonciateurs créent une exception à l'habituel devoir de loyauté des employés envers leur employeur. En contexte gouvernemental, ces dispositions visent bien sûr à prévenir le gaspillage de fonds publics ou d'autres abus de privilèges ou pouvoirs accordés par l'État. En contexte privé $[\ldots]$, leur but conserve un caractère public, puisqu'il s'agit de prévenir les actes répréhensibles «qui constitue[nt] ou [sont] susceptible[s] de constituer une infraction à une loi» $[\ldots]$. L'idée sous-jacente est d'associer les employés à la lutte de l'État contre les conduites illicites, et ce, en leur accordant une certaine immunité contre les représailles des employeurs. ([2005] CSC 70)

Par contre, le point d'équilibre approprié entre la liberté d'expression et le devoir de loyauté est plus difficile à trouver lorsqu'il s'agit de divulguer de l'information à l'externe (c.-à-d. aux agents responsables de l'application de la loi tels que la police, un syndic d'un ordre professionnel, etc.) ou au grand public (c.-à-d. par la voie des médias). Les arbitres et les tribunaux (de droit commun et administratif) ont eu à se pencher sur cette question à de nombreuses reprises au fil des ans $^{14}$. Selon les recherches de Sasseville et Samoisette Fournier (2017), dans un tel cas de figure, « [...] au Canada, il est de jurisprudence constante que la liberté d'expression de l'employé est subordonnée à son devoir de loyauté » (p. 45). Pour mettre à l'écart le devoir de loyauté d'un employé et l'autoriser à faire une divulgation externe ou publique contre son employeur, des conditions exceptionnelles doivent être remplies, à savoir :

1. Avoir épuisé les recours internes;

2. Être de bonne foi; ne pas être motivé par un esprit de vengeance;

3. Divulguer seulement ce qui est d'intérêt public;

4. S'assurer que l'information rendue publique est véridique;

5. S'assurer que l'ampleur de l'intervention publique n'est pas disproportionnée avec l'objectif poursuivi (Sasseville et Samoisette Fournier, 2017, p. 48, citant O'Bomsawin et Marcoux, 2014).

L'importance de la première condition, soit le respect du processus de divulgation interne préalable à toutes divulgations externes et publiques, a été à maintes reprises soulignée par les tribunaux et les arbitres :

[23] [...] Selon une jurisprudence constante et de longue date en matière de relations du travail, on réalise mieux l'équilibre entre ces deux objectifs en encourageant les employés «loyaux » à résoudre les problèmes à l'interne plutôt qu'à s'adresser immédiatement à la police, c'est-à-dire en faisant appel aux mesures internes avant de recourir aux mécanismes publics [...]. (Merk c. Association internationale des travailleurs en ponts, en fer structural, ornemental et d'armature, section locale 771 , [2005] CSC 70) 
Pour être précis quant au principe applicable :

[23] [...] l'obligation de loyauté impose à l'employé d'épuiser les mécanismes internes avant de se prévaloir des «mécanismes publics ». De tels mécanismes internes visent à faire en sorte que la réputation de l'employeur ne soit pas entachée par des accusations injustifiées, s'appuyant sur des renseignements inexacts. (Merk c. Association internationale des travailleurs en ponts, en fer structural, ornemental et d'armature, section locale 771, [2005] CSC 70)

Bref, chaque fois qu'un salarié décide de divulguer publiquement un acte répréhensible de son employeur, il doit être conscient qu'il se met dans une situation précaire, car « le fardeau de démontrer qu'il répond à chacune des conditions énumérées plus haut [les cinq conditions] lui incombe» (Sasseville et Samoisette Fournier, 2017, p. 48). S’il ne réussit pas à prouver ces conditions, le salarié risque d'être accusé de déloyauté, de mauvaise foi, voire de trahison, et de faire l'objet de mesures disciplinaires très sévères.

Or, plusieurs observateurs critiquent cette application étroite du devoir de loyauté vis-à-vis de l'employeur parce que la liberté d'expression n'a pas le même niveau hiérarchique sur le plan juridique que l'obligation de loyauté. En effet, au Canada, la liberté d'expression est un droit qui est prévu dans les chartes des droits, ce qui lui donne un caractère constitutionnel ou quasi constitutionnel, tandis que l'obligation de loyauté est inscrite dans une loi ordinaire, soit le Code civil du Québec ${ }^{15}$ :

Il faut regretter que l'obligation de loyauté demeure ainsi « la règle» et la liberté d'expression, «l'exception», alors que la première devrait plutôt être subordonnée à la seconde, dans le respect de la hiérarchie normative. Dans les faits, la liberté de parole devrait bénéficier d'un préjugé favorable pour ainsi ravaler l'obligation de loyauté au rang d'exception à ce grand principe. Au lieu de se demander si les circonstances justifient une dérogation à l'obligation de loyauté, il faudrait vérifier si elles rendent raisonnablement nécessaire la limitation que l'employeur souhaite apporter à la liberté d'expression du salarié (Brunelle et Samson, 2005, p. 854).
Certains ont démontré que la loyauté est une question complexe et qu'elle se décline en trois dimensions : l'employé doit être loyal 1) à son employeur, mais aussi 2) à lui-même et à ses valeurs et 3) à la société en général (Cailleba, 2019). Or, il ne devrait pas y avoir de contradiction entre ces trois dimensions de la loyauté, si on la recadre avec le concept plus large de «loyauté rationnelle» (rational loyalty; Vandekerkhove et Commers, 2004; Vandekerkhove et Tsahuridu, 2010).

Dans cette ligne de pensée, l'objet de la loyauté n'est pas vis-à-vis des aspects physiques d'une organisation ou d'un employeur (c.-à-d. les bâtiments, les cadres, les conseils d'administration, les collègues, etc.), mais plutôt vis-à-vis de son énoncé de mission, de ses objectifs, de ses valeurs et de son code de conduite. «La loyauté du lanceur d'alerte s'adresse tout à la fois à la mission d'origine de son employeur, comme à la société entière puisque celui-ci ne saurait faire défaut à son engagement pris auprès d'elle» (Cailleba, 2019, p. 250). L'organisation fait partie de la société et contribue à son développement. C'est de cette mission que l'organisation tire sa légitimité :

Near et Miceli [...] ont, en plus, plusieurs fois montré que le souci premier du lanceur d'alerte est de voir corriger ce comportement non éthique afin que son organisation puisse poursuivre sa mission. Le lanceur d'alerte n'a pas la volonté de nuire à son entreprise mais plutôt de lui servir, alors que celle-ci ignore que ce qu'elle fait, ou laisse faire, la dessert à court, moyen ou long terme (Vandekerkhove et Tsahuridu, 2010). (Cailleba, 2019, p. 251)

Or, il nous semble que le concept de loyauté rationnelle trouve une application encore plus forte aux employés du secteur public, car ils sont justement au service du public. Ainsi, «[...] une exigence de loyauté aux gestionnaires plutôt qu'à l'égard du public est de nature à entretenir des pratiques déloyales à l'égard du public», surtout lorsque le bien-être de la collectivité est en jeu (Trudel, 2019, s. p.).

Enfin, si l'employé est un professionnel (au sens du Code des professions du Québec), ses obligations déontologiques l'obligent à tenir compte des conséquences prévisibles de ses travaux, interventions ou recherches à l'égard du public. Tout professionnel doit désormais se demander si ses interventions engendrent des impacts dans son milieu plus ou 
moins immédiat. Il ne lui suffit plus de s'interroger sur leur conformité à une démarche préétablie. Avec une telle obligation déontologique, le professionnel (salarié ou consultant) d'une organisation du secteur privé ou public ne peut se dispenser de pleinement participer à la définition du «développement» de l'organisation, de l'entreprise ou de la société, ni de considérer la contribution (ou les impacts des décisions) des parties prenantes (Simard et Morency, 2010).

\section{L'évolution du droit canadien et québécois en matière de protection du lanceur d'alerte}

Si la divulgation s'intègre dans une longue tradition aux États-Unis ${ }^{16}$ et au Royaume-Uni ${ }^{17}$, cette pratique est plutôt nouvelle au Canada (Biron et Rousseau, 2012). Le statut conféré aux lanceurs d'alerte dans ces pays n'a pas encore d'équivalent dans la législation canadienne et québécoise (Keith, 2017). Comme nous le verrons, les lanceurs d'alerte sont parfois protégés, mais souvent imparfaitement par quelques lois plus ou moins sectorielles.

\subsection{La législation canadienne (fédérale)}

\subsubsection{Le Code criminel}

Le Code criminel fut l'une des premières dispositions législatives à prévoir, au Canada, une protection accrue des lanceurs d'alerte, tant du secteur public que privé, contre les représailles de leur employeur. Cette protection est valable partout au pays. Largement inspiré par l'article 1107 de la Loi SarbanesOxley américaine, l'adoption en 2004 de l'article 425.1 du Code criminel a permis d'ériger en infraction criminelle le fait de prendre ou de menacer de prendre des mesures de représailles contre un employé dans le seul but de l'empêcher de fournir des renseignements reliés à la violation, par l'employeur ou une personne agissant en son nom, d'une disposition du Code criminel ou de toute loi fédérale ou provinciale, incluant les règlements adoptés en vertu de ces lois.

S'il est reconnu coupable de représailles contre un lanceur d'alerte, le contrevenant est passible d'une peine d'emprisonnement maximale de cinq ans. De plus, le paragraphe 425.1(2) prévoit que le contrevenant pourra engager la responsabilité criminelle de son organisation. Cette dernière pourra être condamnée à une amende, dont le Code criminel ne prévoit aucun montant maximal.

\section{Analyse sommaire}

Même si l'adoption de l'article 425.1 du Code criminel était un pas vers la bonne direction, sa portée s'avère encore insuffisante pour plusieurs observateurs, et ce, pour diverses raisons.

La protection de cet article ne s'applique qu'aux employés d'une organisation publique ou privée. Les entrepreneurs indépendants qui ne sont pas des employés au sens de la loi ne seront pas protégés si, après une divulgation, ils subissent des représailles (Bowal, 2011).

Aussi, l'article 425.1 du Code criminel ne s'applique qu'aux actes répréhensibles d'un employeur qui constituent une infraction criminelle ou un acte illégal (p. ex., la corruption, le complot, le délit d'initié et la fraude). Il ne protège donc pas les employés qui divulguent un acte répréhensible qui ne constitue pas une infraction criminelle (p. ex., un abus de pouvoir, une violation d'une politique interne d'une organisation, une mauvaise gestion des ressources financières) ou, de façon plus générale, un comportement contraire à l'éthique et à la déontologie. Par conséquent, avant de dénoncer, un employé doit être certain que cet acte répréhensible est bel et bien un comportement qui constitue une infraction criminelle. Or, à moins d'être un avocat, de bien connaître les législations fédérale et provinciale et d'avoir accès à toutes les preuves disponibles, un employé qui souhaite divulguer de l'information dans un tel contexte difficile court de grands risques (Bowal, 2011).

Enfin, cette protection n'est efficace que lorsque le lanceur d'alerte divulgue des renseignements à un agent dont les attributions comportent le contrôle de l'application d'une loi fédérale ou provinciale. En d'autres termes :

Les révélations reliées à la fraude corporative, à un environnement de travail dangereux ou au non-respect des normes du travail devront être effectuées auprès, respectivement, des autorités policières et de la [Commission des normes, de l'équité, de la santé et de la sécurité du travail]. (Bonhomme et Paquette, 2006, p. 216) 
Un employé loyal envers son employeur et bien intentionné qui divulgue un acte répréhensible à son superviseur, à d'autres supérieurs au sein de l'organisation ou aux médias ne pourra pas bénéficier de la protection de l'article 425.1 (Brunelle et Samson, 2005; Keith, 2017).

Pour toutes ces raisons, peu de décisions judiciaires ont donc été rendues à ce jour ${ }^{18}$. Par contre, le spectre d'une éventuelle poursuite au criminel, d'une peine d'emprisonnement ou d'une amende a peut-être un effet dissuasif sur certains employeurs qui seraient tentés d'utiliser des mesures de représailles après une divulgation d'un employé. Cet état de fait demeure toutefois à prouver.

\subsubsection{La Loi sur la protection des fonctionnaires divulgateurs d'actes répréhensibles (LPFDAR)}

Dans la foulée de l'utilisation frauduleuse des fonds fédéraux destinés à la promotion de l'unité nationale au Québec (scandales des commandites) au début des années 1990, de l'affaire des dépenses abusives du commissaire à la vie privée du Canada George Radwanski en 2003 et de la mauvaise gestion du ministère des Ressources humaines à Ottawa en 2000, le législateur adoptait, en 2005, la Loi sur la protection des fonctionnaires divulgateurs d'actes réprébensibles (LC 2005, chap. 46) ${ }^{19}$. En effet, ces affaires, en plus d'affaiblir considérablement la confiance du public envers les entreprises et le gouvernement, ont vu plusieurs fonctionnaires divulgateurs (expression utilisée dans la loi) payer le prix fort tant sur le plan professionnel que personnel pour avoir pris la parole afin de défendre l'intérêt public.

À titre d'exemple, en 1996, trois ans avant que le scandale des commandites soit dévoilé au grand public, Allan Cuttler, un employé, avait divulgué des pratiques douteuses liées à la gestion des fonds publics du ministère des Travaux publics. Il a subi certaines représailles à la suite de cette plainte, puis a été muté dans un autre département avant de prendre sa retraite (Desjardins et Jutras, 2007). L'année suivante (1997), le gouvernement a créé le Programme des commandites pour accroittre sa visibilité et pour rehausser son image au public. Ce programme était géré par le ministère des Travaux publics. Deux ans plus tard (1999), le journaliste Daniel Leblanc a écrit des articles dans le Globe and Mail sur des pratiques douteuses du gouvernement au pouvoir. Monsieur
Leblanc avait été contacté par plusieurs sources anonymes (nom de code : ma chouette) et avait eu accès à des documents grâce à la Loi sur l'accès à l'information. De plus, cette situation a fait l'objet d'une décision de la Cour suprême du Canada, car une agence de publicité retenue par le gouvernement fédéral dans le cadre du Programme des commandites a voulu forcer un journaliste à lui dévoiler le nom de l'employé qui avait fait la divulgation (Globe and Mail c. Canada Procureur général], [2010] CSC 41, [2010] 2 RCS 592).

\section{Mécanismes de divulgation d'actes réprébensibles}

La LPFDAR, entrée en vigueur en avril 2007, vise à établir un équilibre approprié entre le devoir de loyauté des fonctionnaires envers leur employeur, leur droit à la liberté d'expression, comme le garantit la Charte canadienne des droits et libertés, et l'intérêt du public (préambule de la LPFDAR). Elle donne accès aux personnes employées dans le secteur public fédéral, aux membres de la Gendarmerie royale du Canada ainsi qu'aux administrateurs généraux (y compris les sous-ministres et les premiers dirigeants) à des régimes de divulgation d'actes répréhensibles et de protection contre les représailles ${ }^{20}$. Elle vise la divulgation faite de bonne foi d'actes répréhensibles commis ou à venir au sein du secteur public ou le concernant, soit :

1) une contravention aux lois fédérales ou provinciales;

2) l'usage abusif de fonds ou de biens publics;

3) les cas graves de mauvaise gestion dans le secteur public;

4) les risques graves pour la vie, la santé, la sécurité humaine ou l'environnement;

5) la contravention grave à un code de conduite établi en vertu de la LPFDAR ${ }^{21}$;

6) le fait de sciemment ordonner ou de conseiller à une personne de commettre l'un de ces actes.

Afin d'opérationnaliser les principes des meilleures pratiques en matière de divulgation, la LPFDAR prévoit plusieurs instances (niveaux) de divulgation des actes répréhensibles, soit les mécanismes de divulgation interne, externe et publique.

Les administrateurs généraux doivent, dans leur code de conduite, établir des mécanismes de divulgation interne pour les fonctionnaires faisant partie de l'élément du secteur public (ministères, sociétés d'État, etc.) dont ils sont responsables (art. 10). Ils doivent désigner un agent supérieur chargé de recevoir tous 
les renseignements qui, de l'avis d'un fonctionnaire, relèvent d'un acte répréhensible et d'y donner suite d'une façon compatible avec le code de conduite établi par le Secrétariat du Conseil du Trésor (art. 11). Cette divulgation interne est prioritaire.

Si la question n'est pas réglée correctement à ce palier ou s'il y a lieu de croire que la question ne peut être divulguée de manière confidentielle au sein de son secteur, le fonctionnaire peut la soumettre à l'externe, c'est-à-dire au Commissariat à l'intégrité du secteur public du Canada (CISP), un organisme gouvernemental apte à recevoir les plaintes et les rapports de représailles du divulgateur, et à y enquêter (art. 13). Si le commissaire rejette la plainte, la décision peut faire l'objet d'un contrôle judiciaire à la Cour fédérale $(\operatorname{art} .51 .2)^{22}$.

Enfin, un fonctionnaire peut faire une divulgation publique s'il n'a pas suffisamment de temps pour faire la divulgation, dans la forme prévue, et s'il a des motifs raisonnables de croire que l'acte ou l'omission visés par la divulgation constituent une infraction grave à une loi fédérale ou provinciale, ou encore un risque imminent, grave et précis pour la vie, la santé ou la sécurité humaines ou pour l'environnement (art. 16).

\section{Mécanismes pour la protection des représailles}

La LPFDAR prévoit un régime de protection vis-àvis des représailles effectuées à l'endroit d'un fonctionnaire ou d'un ancien fonctionnaire pour le motif qu'il a fait une divulgation protégée ou qu'il a collaboré de bonne foi à une enquête menée sur la base d'une divulgation (art. 19). Le fonctionnaire qui a des motifs raisonnables de croire qu'il a été victime de représailles peut déposer dans un premier temps une plainte auprès du Commissaire à l'intégrité du secteur public du Canada (art. 19.1). C'est l'article 2 de la LPFDAR qui définit ce qu'il faut entendre par «représailles ». On y inclut des actions telles que :

a) la sanction disciplinaire;

b) la rétrogradation du fonctionnaire;

c) son licenciement et, s'agissant d'un membre de la Gendarmerie royale du Canada, son renvoi ou congédiement;

d) toute mesure portant atteinte à son emploi ou à ses conditions de travail;

e) toute menace à cet égard.
Il appartient au commissaire d'examiner la plainte et de déterminer si elle est recevable ou non. Si la plainte est jugée recevable, le commissaire peut charger une personne d'enquêter sur la plainte (art. 19.7) ${ }^{23}$. Son rapport est soumis au commissaire le plus tôt possible après la fin de l'enquête (art. 20.3).

$\mathrm{Si}$, après réception du rapport d'enquête, le commissaire est d'avis qu'il y a des motifs raisonnables de croire que des représailles ont été exercées à l'égard du plaignant, il peut demander au Tribunal de la protection des fonctionnaires divulgateurs (TPFD) de décider si des représailles ont été exercées à l'égard du plaignant et, le cas échéant, ordonner des mesures de réparation à l'égard du plaignant, voire la prise de sanctions disciplinaires à l'encontre de la personne ou des personnes identifiées dans la demande comme étant celles qui ont exercé les représailles (art. 20.4) ${ }^{24}$.

\section{Analyse sommaire}

Plusieurs critiques ont fait remarquer que la LPFDAR n'a pas donné tous les résultats attendus depuis son adoption en 2007 (Bron, 2019; Ferguson, 2018; Martin-Bariteau et Newman, 2018; Shepherd, 2017; Keith, 2017; Lévesque, 2014). L'organisation Transparency International et des syndicats québécois ont fait plusieurs observations à cet effet devant la Commission d'enquête sur l'octroi et la gestion des contrats publics dans l'industrie de la construction (Charbonneau et Lachance, 2015, tome 1; Wolfe et collab., 2014). Le Comité permanent des opérations gouvernementales et des prévisions budgétaires (2017; ci-après appelé "Comité permanent») a décidé, à la demande du président du Conseil du Trésor, de réaliser le premier examen législatif de la LPFDAR en février 2017. Dans le cadre de son étude, le Comité permanent a tenu 12 réunions, entendu 52 témoins et reçu 12 mémoires sur le sujet. Le rapport propose 15 recommandations visant à améliorer les objets et les processus de la LPFDAR afin de garantir l'intégrité du secteur public et la protection des divulgateurs canadiens.

En comparaison avec l'article 425.1 du Code criminel, la portée des actes répréhensibles qui peuvent être divulgués est plus large, ainsi que l'entité ou la personne à qui l'employé peut faire la divulgation, en vertu de la LPFDAR (Keith, 2017). Par contre, la loi 
fédérale ne prévoit aucune protection pour les divulgateurs (lanceurs d'alerte) travaillant dans le secteur privé, « ni pour les personnes qui ont aidé les divulgateurs, les témoins et ceux pris à tort comme étant des divulgateurs» (Comité permanent, 2017, p. 71). On soulève aussi que les auteurs de représailles peuvent trop souvent échapper à la punition sous le régime de la LPFDAR en recherchant rapidement un emploi dans le secteur privé. Ce problème est aggravé par le fait que «le Commissaire [à l'intégrité du secteur public du Canada] ne divulgue pas l'identité des contrevenants, ce qui signifie que les employeurs potentiels ne sont pas au courant d'une faute antérieure» (Keith, 2017, p. 282). Par ailleurs, le degré de gravité requis pour qu'un acte soit considéré comme répréhensible est souvent difficile à quantifier. Qu'est-ce qui distingue, par exemple, les cas graves des cas simples de mauvaise gestion? Cette distinction est importante, car elle déterminera si une divulgation est protégée ou non par les mécanismes de la LPFDAR.

De façon plus générale, l'efficacité de certains aspects du processus de plainte pour les fonctionnaires divulgateurs est remise en question dans le rapport du Comité permanent. Même s'il y a plusieurs plaintes au commissaire, peu ont fait l'objet d'une enquête au fil des ans et encore moins donnent raison aux fonctionnaires divulgateurs selon Me Paul Lalonde de Transparency International (Lévesque, 2014). Tous les ans, le Secrétariat du Conseil du Trésor (SCT) du Canada doit produire un rapport annuel conformément à l'article 38.1 de la LPFDAR. À titre d'exemple, dans le cadre du rapport annuel 2019-2020, sur 116 plaintes respectant la définition d'un acte répréhensible, 38 ont mené à une enquête, et seulement 3 ont donné raison au divulgateur et ont nécessité des mesures correctives (SCT, 2020).

Par ailleurs, le SCT (2020) mentionne qu'un sondage est réalisé tous les ans sur l'éthique en milieu de travail auprès des fonctionnaires fédéraux. Un élément attire l'attention: $50 \%$ des répondants affirment ne pas avoir suffisamment confiance au système pour amorcer un processus de recours officiel sans crainte de représailles. De ce fait, malgré l'existence de cette loi, la divulgation (interne) d'un acte répréhensible par des employés reste difficile ${ }^{25}$.

Certains considèrent que les pouvoirs du Tribunal de la protection des fonctionnaires divulgateurs sont plutôt limités lorsque vient le temps de trouver la mesure de réparation la plus indiquée aux circonstances particulières d'un dossier : annuler les actions préjudiciables, réintégrer un employé ou verser une compensation (art. 21.7). Or, il peut arriver que «les représailles subies par le plaignant ne peuvent être facilement réduites à une valeur pécuniaire (si, par exemple, l'employé a été harcelé ou a raté des occasions de promotion), alors il ne sait pas comment le Tribunal pourrait élaborer une réparation appropriée» (Ferguson, 2018, p. 1049, trad. libre).

\subsubsection{Autres lois sectorielles au fédéral}

De façon plus sectorielle, la protection de la divulgation d'information se retrouve au fédéral dans quelques lois, soit la Loi canadienne sur la protection de l'environnement (LC 1999, chap. 33), le Code canadien du travail (LRC 1985, chap. L-2) et la Loi sur la protection des renseignements personnels et les documents électroniques (LC 2000, chap. 5).

\subsection{La législation québécoise (provinciale)}

La législation et la réglementation québécoises traitant de la divulgation d'actes répréhensibles et de la protection des lanceurs d'alerte sont, principalement, dans le Code civil du Québec, la Loi concernant la lutte contre la corruption, la Loi facilitant la divulgation d'actes réprébensibles dans les organismes publics, le Règlement 52-110 sur le comité d'audit adopté par l'Autorité des marchés financiers et la Loi sur l'encadrement $d u$ secteur financier. On trouve aussi certaines dispositions dans d'autres lois plus sectorielles. Il convient de ne pas négliger les recommandations de la commission Charbonneau en faveur d'un régime général de protection, lequel se trouve fréquemment à inspirer la législation québécoise.

\subsubsection{Le Code civil du Québec}

La réforme du Code civil du Québec, entrée en vigueur en 1994, a introduit une disposition générale permettant à toute personne de se dégager de sa responsabilité civile pour le préjudice causé à autrui à la suite de la divulgation d'un secret commercial lorsqu'elle prouve que l'intérêt général l'emporte sur le maintien du secret et, notamment, que la divulgation de celuici est justifiée par des motifs de santé ou de sécurité du public (art. 1472). «Il [cet article] s'adresse tout particulièrement aux situations qui touchent la libre circulation de produits très nocifs pour le public, ainsi que celles où la découverte d'un produit très efficace 
afin de combattre une maladie grave est cachée du public » (Karim, 2020, s. p.). En effet, le législateur a voulu éviter qu'en raison de seules considérations financières, ce type d'information vitale pour la santé et la sécurité d'une collectivité soit caché.

\section{Analyse sommaire}

Cette protection des divulgateurs (lanceurs d'alerte) offerte par le Code civil du Québec est ouverte pour les salariés du secteur public et privé, mais aussi pour toute autre personne qui veut divulguer une information. Par contre, cette exonération de responsabilité est ouverte seulement à une personne qui divulgue un secret commercial, notion que le législateur n'a pas définie (Karim, 2020, s. p.).

De plus, la protection de cette disposition est très limitée. Elle protège « un lanceur d'alerte uniquement contre les poursuites civiles et non contre les autres mesures de représailles. Il n'est donc pas à l'abri d'une perte d'emploi, d'une rétrogradation, d'une mutation ou d'un départ forcé» (Charbonneau et Lachance, 2015, tome 1, p. 47).

Enfin, cette disposition semble très peu utilisée devant les tribunaux jusqu’à présent.

\subsubsection{La Loi concernant la lutte contre la corruption (LCLCC)}

Au Québec, les allégations de pratiques douteuses liées à l'octroi, par des organismes publics, de contrats de travaux publics, de services ou d'approvisionnement se sont multipliées dans les médias en 2007. Ces allégations ont notamment visé les conflits d'intérêts dans l'octroi du contrat des compteurs d'eau par la Ville de Montréal, la vente sans aucun profit par la Ville de Montréal des terrains à la base du projet immobilier Faubourg Contrecœur, les pratiques de favoritisme du Fonds de solidarité FTQ en faveur d'un des plus gros entrepreneurs en construction du Québec, un stratagème de collusion par truquage d'offres et partage de contrats, des pratiques de financement politique illégal, de l'évasion fiscale et des fausses facturations.

Devant une situation de plus en plus décriée par le public, le gouvernement du Québec a créé par décret, en février 2011, l'Unité permanente anticorruption (UPAC), une organisation ayant pour mission de coordonner et de diriger les forces et expertises en place au sein du gouvernement pour lutter contre la corruption, la collusion et le trafic d'influence dans l'adjudication et l'exécution de contrats publics, particulièrement dans l'industrie de la construction ${ }^{26}$. La création de l'UPAC, inspirée du Department of Investigation de la Ville de New York, était une première au Canada.

Un peu plus tard dans la même année, le Québec adoptait la Loi concernant la lutte contre la corruption (RLRQ, chap. L-6.1), ayant pour objet de renforcer les actions de prévention et de lutte contre la corruption en matière contractuelle dans le secteur public, et de contribuer à favoriser la confiance du public dans les marchés publics (art. 1). À cette fin, le législateur a voulu préciser la structure, les pouvoirs et le fonctionnement de l'UPAC, et instituer la charge de commissaire à la lutte contre la corruption, dont la principale mission est de diriger l'UPAC.

Dans la foulée, la LCLCC établit, d'une part, une procédure facilitant la dénonciation (terme utilisé dans cette loi) des actes répréhensibles très ciblés (soit la corruption, la collusion, la fraude, la malversation et le trafic d'influence) en lien avec un contrat dans le secteur public québécois ${ }^{27}$ et, d'autre part, un mécanisme de protection contre les représailles $^{28}$. À l'instar de scandales à l'échelle fédérale, les lanceurs d'alerte ont dû, eux aussi, payer le prix fort sur les plans professionnel et personnel pour avoir lancé une alerte publique. À titre d'exemple, prenons Ken Pereira, qui a dénoncé les différentes situations dont il avait été témoin avec des dirigeants du Fonds de solidarité FTQ dans le cadre de ses fonctions professionnelles. Son identité n'a pas fait l'objet d'une protection lorsqu'il a commencé à partager ses expériences en dehors des strictes relations contractuelles divulguées. La LCLCC ne protège pas les lanceurs d'alerte (qualificatif attribué par la commission Charbonneau) qui mettent au jour un contexte de corruption d'ordre général.

Sur le plan pratique, une personne désirant faire une dénonciation doit recourir à une procédure assez exigeante. Elle doit communiquer au Commissaire à la lutte contre la corruption tout renseignement pouvant démontrer qu'un acte répréhensible a été commis ou est sur le point de l'être (art. 26). Le commissaire doit prendre toutes les mesures nécessaires afin de s'assurer que l'anonymat du dénonciateur soit préservé (art. 31). 
Après analyse, le commissaire peut refuser de donner suite à une dénonciation, s'il estime qu'elle est frivole ou ne relève pas de sa mission. Dans ce cas, il en informe le dénonciateur.

S'il accepte de donner suite à la dénonciation, le commissaire transmet le dossier, selon le cas, à un commissaire associé ou aux équipes d'enquête concernées (art.29). Signalons que le dénonciateur d'un acte répréhensible peut faire cette dénonciation malgré la Loi sur l'accès aux documents des organismes publics et sur la protection des renseignements personnels (RLRQ, chap. A-2.1) ou la Loi sur la protection des renseignements personnels dans le secteur privé (RLRQ, chap. P-39.1). De plus, il peut faire une dénonciation malgré toute obligation de loyauté ou de confidentialité pouvant le lier, notamment à l'égard de son employeur ou de son client (art. 27). Toutefois, la LCLCC ne permet pas de communiquer des renseignements protégés par le secret professionnel liant l'avocat ou le notaire à son client (art. 27).

La LCLCC prévoit aussi l'interdiction d'exercer des représailles (rétrogradation, suspension, congédiement, déplacement, sanction disciplinaire ou toute autre mesure portant atteinte à son emploi ou à ses conditions de travail) à l'encontre d'une personne à l'origine de la dénonciation, de menacer cette personne de représailles pour qu'elle s'abstienne de faire une dénonciation ou de collaborer à une telle vérification ou enquête (art. 32 et 33). Quiconque contrevient à cette interdiction est passible d'une amende sévère ${ }^{29}$.

Afin de protéger les salariés qui dénoncent un acte répréhensible commis dans leur organisation ou qui collaborent à une vérification ou enquête portant sur un tel acte, la LCLCC a modifié la Loi sur les normes du travail (LNT; RLRQ, chap. N-1.1) ${ }^{31}$. D'une part, la LNT [parag. 122(7) et art. 123] prévoit la possibilité de déposer une plainte pour un dénonciateur salarié contre son employeur (privé et public) devant la Commission des normes, de l'équité, de la santé et de la sécurité du travail (CNESST) ${ }^{31}$.

Lorsque le salarié syndiqué dépose un grief, si la plainte est jugée recevable par la CNESST, cette dernière offre des services de médiation. Si aucun règlement n'intervient entre les parties, la CNESST défère alors la plainte au Tribunal administratif du travail [parag. 123.4(1) de la LNT]. Cette dernière prévoit même une présomption simple en faveur du salarié. En effet, si le salarié établit à la satisfaction du Tribunal que la sanction lui a été imposée ou que la mesure a été prise contre lui à cause de l'exercice de ce droit, il incombe alors à l'employeur de prouver qu'il a pris cette sanction ou mesure à l'égard du salarié pour une autre cause juste et suffisante [paragr. 123.4(2) de la LNT et art. $17 \mathrm{du}$ Code du travail (RLRQ, chap. C-27)].

\section{Analyse sommaire}

La LCLCC institue une procédure permettant la dénonciation à l'UPAC d'actes répréhensibles très ciblés au sujet d'un contrat public. Le dénonciateur sera sans protection s'il dénonce une situation plus générale. De plus, «la LCLCC ne prévoit aucune protection pour les lanceurs d'alerte qui s'adressent directement à un supérieur hiérarchique, à un collègue ou à un responsable d'organisme public autre que l'UPAC pour dénoncer un acte répréhensible » (Charbonneau et Lachance, 2015, tome 3, p. 110).

Pour couvrir les situations d'ordre plus général, il a fallu attendre la Loi facilitant la divulgation d'actes répréhensibles à l'égard des organismes publics pour que les divulgateurs/dénonciateurs puissent faire l'objet d'une protection contre les représailles.

\subsubsection{La Loi facilitant la divulgation d'actes répréhensibles à l'égard des organismes publics (LFDAROP)}

En plus d'adopter la LCLCC, le gouvernement du Québec est allé encore plus loin en créant, en novembre 2011, par décret, la Commission d'enquête sur l'octroi et la gestion des contrats publics dans l'industrie de la construction (commission Charbonneau) ${ }^{32}$. Cette commission avait pour mission d'examiner l'existence de stratagèmes et, le cas échéant, de brosser un portrait de ceux qui participent à des activités de collusion et de corruption dans l'octroi et la gestion de contrats publics dans l'industrie de la construction ainsi que des liens possibles avec le financement des partis politiques. Le gouvernement du Québec a aussi demandé à la commission Charbonneau de mettre au jour les possibles activités d'infiltration de l'industrie de la construction par le crime organisé et, enfin, d'examiner des pistes de solution et de faire des recommandations en vue d'établir des mesures permettant d'identifier, d'enrayer et de prévenir ces stratagèmes et activités d'infiltration. La 
commission a commencé ses audiences le 22 mai 2012 et a déposé son volumineux rapport en novembre 2015.

Parmi ses recommandations au gouvernement, la commission Charbonneau suggère de créer un régime général de protection pour l'ensemble des lanceurs d'alerte de tous les secteurs (public et privé), mais aussi pour élargir le spectre des actes répréhensibles qui peuvent faire l'objet d'une divulgation :

Afin de favoriser une plus grande participation des citoyens à l'assainissement des pratiques contractuelles relatives aux infrastructures publiques, au financement des partis politiques liés à ces pratiques, et à l'infiltration de l'industrie de la construction par le crime organisé, la Commission est d'avis qu'un régime général de protection des lanceurs d'alerte s'impose. Un tel régime permettrait non seulement d'assurer la protection de tous les lanceurs d'alerte, mais aussi de leur offrir l'accompagnement et le soutien requis, notamment au plan financier. C'est qu'en dépit des recours à leur portée, les lanceurs d'alerte peuvent avoir à engager des dépenses importantes pour faire valoir leurs droits, notamment lorsqu'ils font face à de grandes organisations aux ressources financières importantes, qui ont la capacité de mener une lutte judiciaire de longue durée. (Charbonneau et Lachance, 2015, tome 3, p. 110)

Pour donner une suite aux recommandations de la commission Charbonneau, l'Assemblée nationale a adopté, en décembre 2016, le Projet de loi no 87, Loi facilitant la divulgation d'actes répréhensibles dans les organismes publics (RLRQ, chap. D-11.1), qui est entrée en vigueur le $1^{\text {er }}$ mai 2017. Cette loi (LFDAROP), comme son titre l'indique, a pour mission de faciliter la divulgation (terme utilisé par la Loi) d'actes répréhensibles dans les organismes publics québécois tels que les ministères, les commissions scolaires (devenues, depuis juin 2020, des centres de services scolaires), les entreprises du gouvernement (p. ex., Hydro-Québec et la Caisse de dépôt et placement du Québec), les universités et les établissements publics de santé et de services sociaux, puis d'établir un régime de protection contre les représailles (art. 1 et 2). Il est important de remarquer que les municipalités étaient exclues au départ du champ d'application de la LFDAROP.
«Cette situation était surprenante dans le contexte où les malversations les plus importantes révélées à la CEIC [commission Charbonneau] ont eu lieu dans des municipalités »(Russell, 2019, p. 99). En octobre 2018, la LFDAROP a été modifiée afin que les lanceurs d'alerte municipaux puissent bénéficier de cette protection législative, mais sous certaines conditions.

Au sens de la LFDAROP est considéré comme répréhensible tout acte qui constitue, selon le cas :

1 une contravention à une loi du Québec ou à une loi fédérale applicable au Québec;

2) un manquement grave aux normes d'éthique et de déontologie;

3) un usage abusif des fonds ou des biens d'un organisme public, y compris de ceux qu'il gère ou détient pour autrui;

4) un cas grave de mauvaise gestion au sein d'un organisme public;

5) le fait, par un acte ou une omission, de porter gravement atteinte ou de risquer de porter gravement atteinte à la santé ou à la sécurité d'une personne ou à l'environnement (art. 4).

Sur le plan pratique, la LFDAROP prévoit que «toute personne peut, en tout temps, divulguer [...] des renseignements pouvant démontrer qu'un acte répréhensible a été commis ou est sur le point de l'être à l'égard d'un organisme public [...]» (art. 6).

Selon Sasseville et Samoisette Fournier (2017) :

La Loi sanctionnée est ouverte à toute personne, ce qui peut inclure un employé autant qu'un usager des services publics, un journaliste, un syndicat, un cocontractant du gouvernement, un entrepreneur ayant déposé une proposition dans le cadre d'un appel d'offres, etc. (p. 56)

La même disposition prévoit que la personne qui procède à cette divulgation peut le faire en s'adressant soit au Protecteur du citoyen, soit à un responsable du suivi des divulgations nommé au sein de son organisme public. À cet effet, une procédure pour faciliter la divulgation d'actes répréhensibles par les employés devra être établie et diffusée au sein de chaque organisme public par la personne ayant la plus haute autorité administrative (art. 18) $)^{33}$. 
Une personne peut aussi faire une divulgation publique, notamment par la voie des médias, si elle a des motifs de croire que cet acte répréhensible commis ou sur le point de l'être présente un risque grave pour la santé ou la sécurité d'une personne ou l'environnement, et qu'elle ne peut pas, compte tenu de l'urgence de la situation, s'adresser au Protecteur du citoyen ou à un responsable des divulgations. Avant de procéder à cette divulgation publique, la personne en question devra communiquer ces renseignements à la police ou au Commissaire à la lutte contre la corruption (soit l'UPAC) (art. 7).

La LFDAROP n'oblige pas le fonctionnaire ou l'employé témoin d'un acte répréhensible à le divulguer. Elle laisse à la discrétion du témoin le soin de le faire sur la base d'une simple obligation morale. En vertu d'une obligation de loyauté accrue, seuls les membres de la direction d'un ministère ou d'un organisme ont le devoir de divulguer une situation de corruption dont ils seraient témoins. Cette obligation découle d'un jugement de la Cour suprême du Canada dans l'affaire Banque de Montréal c. Kuet Leong Ng, [1989] 2 RCS 429.

La LFDAROP interdit, sous peine d'amendes, les représailles ou des menaces de représailles à l'encontre d'une personne qui divulgue une vérification ou une enquête menée à la suite d'une divulgation, ou qui y collabore (art. 26). Sont présumés être des représailles la rétrogradation, la suspension, le congédiement ou le déplacement ainsi que toute autre mesure disciplinaire ou mesure portant atteinte à son emploi ou à ses conditions de travail (art. 31). Quiconque contrevient à cette obligation commet une infraction et est passible d'une amende de $2000 \$$ à $20000 \$$ dans le cas d'une personne physique ou, dans tous les autres cas, d'une amende de $10000 \$$ à $250000 \$($ art. 33) 34 .

\section{Analyse sommaire}

Contrairement aux recommandations de la commission Charbonneau, le régime ne protège pas la divulgation d'actes répréhensibles dans le secteur privé. Par contre, le régime a une portée plus large quant aux personnes qui peuvent bénéficier de la protection de la loi. De plus, le spectre des actes répréhensibles qui peuvent faire l'objet d'une divulgation se compare à ceux de la LPFDAR adoptée au fédéral au milieu des années 2000.
L'efficacité de la LFDAROP a fait l'objet de plusieurs critiques après le congédiement du lanceur d'alerte Louis Robert, agronome au ministère de l'Agriculture, des Pêcheries et de l'Alimentation du Québec (MAPAQ). Revenons sur la chronologie des événements. En octobre 2017, le divulgateur exposait une situation en vertu de la LFDAROP à la personne responsable du suivi des divulgations de son ministère. À l'époque, monsieur Robert dénonçait l'implication et l'ingérence des entreprises privées dans la recherche publique sur l'usage des pesticides en agriculture et en culture des grains. Selon lui, les entreprises privées ne peuvent intervenir dans les recherches qui guident le gouvernement dans ses prises de décision (interne), puis vendre des produits aux agriculteurs en vertu desdits résultats (externe). Devant l'inaction de son employeur, le divulgateur a transmis des documents confidentiels à Radio-Canada.

En janvier 2019, monsieur Robert a été congédié à la suite des fuites journalistiques. Rapidement, le Protecteur du citoyen, qui a pour mission de veiller au respect des droits des personnes dans la sphère publique et aussi de traiter les actes répréhensibles à l'égard des organisations publiques, a décidé de faire une enquête sur l'application de la LFDAROP par le MAPAQ. De plus, le Protecteur du citoyen voulait se pencher sur les allégations déposées par monsieur Robert à la personne responsable du suivi des divulgations du MAPAQ. Toutefois, quelques jours plus tard, le ministre du MAPAQ demandait à l'organisation précitée de faire une enquête sur le congédiement du divulgateur et du traitement qu'avait reçu sa divulgation datant de la fin de 2017.

En juillet 2019, monsieur Robert a réintégré son ancien poste au sein du MAPAQ, comme il l'avait demandé, avec une entente confidentielle à l'égard de son salaire perdu ${ }^{35}$. Ce dénouement faisait écho à la publication du rapport très critique du Protecteur du citoyen à l'encontre du MAPAQ. Dans ce rapport, il a mis au jour de nombreux problèmes de gestion de la loi (violation de confidentialité de l'identité du lanceur d'alerte, système de circulation non sécurisé de messages électroniques au sein du Ministère, conflit de loyauté, etc.).

Quelques mois plus tard, soit en décembre 2019, un Bilan du Protecteur du citoyen sur la mise en couvre de la Loi facilitant la divulgation d'actes répréhensibles à l'égard des organismes publics a été déposé publiquement. 
Dans le cadre de cette démarche, tout le processus de divulgation a été analysé ainsi que les rôles et responsabilités des parties prenantes. Au total, 21 recommandations ont été soumises par le Protecteur du citoyen. Les premières recommandations concernaient l'élargissement des organisations publiques (p. ex., les filiales de Loto-Québec et d'Hydro-Québec, la Société québécoise du cannabis) et des actes concernés (p. ex., cas de mauvaise gestion, actes ou omissions graves). Les moyens de communication entre les parties concernées par une divulgation ont été précisés, en plus de la préservation de l'anonymat du divulgateur. Une meilleure promotion de cette loi a également été recommandée.

En mars 2020, le Secrétariat du Conseil du trésor (SCT) du Québec a publié un Rapport sur la mise en cuure de la loi. Ce rapport reprend les recommandations du bilan précédemment déposé publiquement. Trois grands objectifs sont visés, soit :

1) revoir le rôle et les responsabilités des acteurs sollicités dans l'application de la LFDAROP;

2) accroître l'efficience de l'application de la LFDAROP; et

3) clarifier certaines dispositions ainsi que créer un comité multipartite (ministère du Travail, de l'Emploi et de la Solidarité sociale, Protecteur du citoyen et Secrétariat du Conseil du trésor) pour analyser, dans son ensemble, le régime de protection contre les représailles afin de trouver des avenues pour en améliorer l'efficience (SCT, 2020).

Le SCT tient également compte de la recommandation du Protecteur du citoyen émise dans le cadre de l'analyse de la situation de monsieur Robert et le MAPAQ. Cette recommandation stipulait :

R-2 Dans le cadre du rapport sur la mise en œuvre de la LFDAROP que doit déposer le président du Conseil du trésor à l'Assemblée nationale au plus tard le 1 er juin 2020, de revoir, plus particulièrement, le rôle et l'encadrement législatif des responsables du suivi des divulgations, afin d'assurer un accompagnement adéquat des lanceurs d'alerte, la confidentialité complète ainsi qu'un traitement des divulgations dépourvu de conflits de loyauté (Protecteur du citoyen, 2019 a, p. 28).

\subsubsection{Le Projet de loi no 198, Loi facilitant la divulgation d'actes répréhensibles (LFDAR)}

En mai 2019, soit un an avant le dépôt du rapport du SCT et du bilan du Protecteur du citoyen, le député de Rosemont élu sous la bannière de Québec solidaire, Vincent Marissal, a proposé le Projet de loi no 198, Loi facilitant la divulgation d'actes réprébensibles (LFDAR). Ce projet de loi visait entre autres à créer un nouveau régime général de protection contre les représailles qui s'appliquerait tant au secteur public que privé et, par ailleurs, à intégrer les dispositions de la nouvelle LFDAR dans des lois telles que celles sur l'administration fiscale, sur l'autorité des marchés publics, sur l'éthique et la déontologie en milieu municipal, sur les normes du travail ainsi que sur les services de garde éducatifs et à l'enfance. Toutefois, le projet de loi no 198 est mort au feuilleton.

\subsubsection{Le Projet de loi no 697, Loi visant à renforcer la divulgation d'actes répréhensibles et la protection des lanceurs d'alerte}

Finalement, le 17 mars 2021, le député de Jonquière, Sylvain Gaudreault, a déposé devant l'Assemblée nationale, afin qu'elle en amorce l'étude, le Projet de loi no 697, Loi visant à renforcer la divulgation d'actes réprébensibles et la protection des lanceurs d'alerte. Essentiellement, ce projet de loi propose de modifier la LFDAROP sur les points suivants :

- élargir la portée de la loi actuelle à d'autres organismes, notamment à ceux qui sont liés à un organisme public par entente contractuelle;

- donner la responsabilité au Protecteur du citoyen de traiter toute divulgation faite par un tiers, mais également par les membres du personnel des organismes visés;

- renforcer la protection des lanceurs d'alerte en affirmant le caractère confidentiel de l'identité de la personne qui fait la divulgation;

- permettre la divulgation au public sans devoir au préalable communiquer avec un corps de police ou le Commissaire à la lutte contre la corruption;

- prévoir que le ministre doit, au plus tard le $1^{\text {er }}$ janvier 2023, et par la suite tous les cinq ans, faire au gouvernement un rapport sur la mise en œuvre de la loi et sur l'opportunité de la maintenir en vigueur ou de la modifier. 
Le projet de loi propose aussi de modifier la Loi sur le Protecteur du citoyen (RLRQ, chap. P-32), afin d'instituer, au sein du Protecteur du citoyen, le Fonds d'aide aux personnes qui effectuent des divulgations d'actes répréhensibles afin d'assurer le financement de leurs frais judiciaires si elles sont victimes de représailles.

Il reste de nombreuses étapes (consultations en commission parlementaire, adoption du principe des députés, étude détaillée en commission parlementaire, prise en considération du rapport de la commission, adoption du projet de loi) avant que le projet de loi no 697 soit sanctionné par le lieutenantgouverneur. Son contenu pourrait être considérablement modifié tout au long du processus.

\subsubsection{Le Règlement 52-110 sur le comité d'audit}

En 2003, les Autorités canadiennes en valeurs mobilières (ACVM), après de vastes consultations ayant pour assise les dispositions de la Loi SarbanesOxley américaine ${ }^{36}$, recommandent d'adopter une série de règlements et de directives visant à augmenter la qualité et l'intégrité de la divulgation d'information des sociétés qui ont émis des titres et qui font un appel public à l'épargne (émetteurs assujettis). Certains de ces règlements et directives seraient obligatoires, d'autres pas.

Puisque la réglementation des marchés financiers est de la compétence des provinces, l'Autorité des marchés financiers (AMF) au Québec a adopté, en 2005, le Règlement 52-110 sur le comité d'audit (RLRQ, chap. V-1.1, r. 28), lequel exige que tout émetteur assujetti se dote d'un comité d'audit chargé d'évaluer objectivement la justesse, entre autres, des pratiques des dirigeants en matière de comptabilité et de communication de l'information financière. Ce comité doit établir des procédures concernant l'envoi confidentiel, sous le couvert de l'anonymat, par les salariés de l'émetteur de préoccupations touchant des points discutables en matière de comptabilité ou d'audit [alinéa $2.3(7)$ b) du règlement].

\subsubsection{La Loi sur l'encadrement du secteur financier (LESF)}

Par ailleurs, en juin 2016, l'AMF, dans l'exercice de ses pouvoirs généraux, a mis sur pied le Programme de dénonciation (lanceurs d'alerte) afin de recueillir de l'information inédite sur des infractions aux lois et règlements qu'elle administre, c'est-à-dire au droit québécois des valeurs mobilières (AMF, 2017, p. 20) 37. Un «dénonciateur» (selon la terminologie de l'AMF) est une personne qui a un accès stratégique à des informations privilégiées. Il peut s'agir d'un employé actuel ou ancien, d'un administrateur, d'un collègue de la personne dont les agissements sont visés, d'un prestataire de services ou d'un proche. Selon l'AMF, ce programme vise à offrir aux dénonciateurs des conditions optimales de protection en matière de confidentialité, d'immunité et de mesures antireprésailles. «Par contre, aucun amendement législatif [dans la Loi sur l'Autorité des marchés financiers] visant à garantir ce qui précède n'avait été mis de l'avant à ce jour» (Mayr et Struthers, 2017, s. p.). Concrètement, le Programme de dénonciation a un certain succès. Dans son rapport annuel 2019-2020, l'AMF (2020) révèle que le Centre d'information de l'AMF a reçu 2765 plaintes et déclarations de pratiques douteuses ou frauduleuses, et en a traité 2568 .

En juin 2018, l'Assemblée nationale a modifié (et ajouté) plusieurs articles de la Loi sur l'Autorité des marchés financiers (RLRQ, chap. A-33.2), qui porte désormais le nom de Loi sur l'encadrement du secteur financier (RLRQ, chap. E-6.1). La section 2 de cette loi vient renforcer la protection des personnes de bonne foi qui souhaitent faire une dénonciation auprès de l'Autorité des marchés financiers. L'article 17.0.1 vise à encourager toute personne qui peut démontrer un manquement réel ou potentiel aux lois que l'AMF est chargée d'appliquer ${ }^{38}$. La préservation de l'anonymat du dénonciateur et l'interdiction d'exercer des mesures de représailles contre celui-ci sont des éléments explicités au articles 17.0.3 à 17.0.5.

Il est important de souligner que la LESF prévoit que :

La personne qui effectue la dénonciation d'un manquement peut le faire malgré la Loi sur l'accès aux documents des organismes publics et sur la protection des renseignements personnels (chapitre A-2.1), la Loi sur la protection des renseignements personnels dans le secteur privé (chapitre P-39.1), toute autre restriction de communication prévue par d'autres lois du Québec, toute disposition d'un contrat ou toute obligation de loyauté ou de confidentialité pouvant la lier, notamment à l'égard de son employeur ou de son client. (art. 17.0.1) 
Elle permet la dénonciation malgré le secret professionnel, sauf pour celui liant les avocats et les notaires à leur client. Cette dernière mesure est sévèrement critiquée par plusieurs ordres professionnels vu l'importance de protéger adéquatement le secret professionnel dans le but d'assurer la protection du public. Certains ordres se sont même tournés vers les tribunaux pour faire déclarer inconstitutionnelle cette nouvelle disposition. Bref, la LESF vient renforcer un volet central de la protection offerte aux personnes qui ont recours au Programme de dénonciation de l'AMF.

En Ontario, le Programme de dénonciation de la Commission des valeurs mobilières de cette province encourage la dénonciation au moyen de récompenses pécuniaires, tandis que l'Autorité des marchés financiers du Québec refuse d'aller dans cette voie. " [L'AMF] prétend que la protection de la confidentialité, et non la perspective d'une récompense financière, demeure la source de motivation première chez le dénonciateur » (RobitailleRodriguez, 2017, s. p.).

\subsubsection{La Loi sur 1'Autorité des marchés publics (LAMP)}

La première recommandation du rapport de la commission Charbonneau en vue d'établir des mesures permettant d'identifier, d'enrayer et de prévenir la collusion et la corruption dans l'octroi et la gestion des contrats publics dans l'industrie de la construction portait sur la création d'une instance nationale d'encadrement des marchés publics.

Pour faire suite à cette recommandation, le gouvernement du Québec a adopté et sanctionné, en décembre 2017, la Loi favorisant la surveillance des contrats des organismes publics et instituant l'Autorité des marchés publics (LQ 2017, chap. 27). Cette loi prévoit la création, depuis mai 2019, de l'Autorité des marchés publics (AMP) et de l'adoption de sa loi constitutive, la Loi sur l'Autorité des marchés publics (chap. A-33.2.). L'AMP a désormais des pouvoirs d'intervention, de vérification et d'enquêtes en ce qui concerne l'adjudication et l'attribution des contrats publics au Québec (p. ex., contrats de gré à gré, demandes de soumission, recommandations pour un contrat conclu à la suite d'un appel d'offres public). Plus précisément, l'AMP a le pouvoir de délivrer des autorisations de contracter avec l'État, de traiter les plaintes des soumissionnaires en matière d'octroi de contrats publics ainsi que de gérer les renseignements et les divulgations en matière contractuelle.

Ainsi, la Loi sur l'Autorité des marchés publics (LAMP) prévoit une protection pour les personnes de bonne foi qui souhaitent communiquer à l'AMP des renseignements relatifs, entre autres, à un processus d'adjudication ou d'attribution d'un contrat public, ou à l'exécution d'un tel contrat, lorsque l'organisme public concerné (p. ex., ministère, municipalité) n'apparaît pas agir ou avoir agi, à l'égard de ce processus ou de ce contrat, en conformité avec le cadre normatif (art. 56). Cette communication est permise sans égard aux dispositions légales restreignant normalement la communication de renseignements à des tiers, notamment le secret professionnel (sauf celui de l'avocat ou du notaire), ou à l'obligation de confidentialité ou de loyauté d'un employé envers son employeur (art. 56).

La préservation de l'anonymat du lanceur d'alerte et l'interdiction d'exercer des mesures de représailles contre celui-ci sont des éléments explicités aux articles 61 à 66 de la LAMP.

\subsubsection{Autres lois sectorielles au Québec}

De façon plus sectorielle, la protection de la divulgation d'information se retrouve dans quelques lois du Québec ayant pour mission de protéger l'environnement, les personnes vulnérables, etc.

\subsubsection{Toutes les lois et les règlements relevant du ministère de l'Environnement et de la Lutte contre les changements climatiques (MELCC)}

En 2005, le MELCC a adopté une Politique ministérielle sur le traitement des plaintes à caractère environnemental. Cette politique permet à tout citoyen témoin d'une situation de signaler une activité pour laquelle il y a lieu de croire à la non-conformité aux lois et aux règlements administrés par ce ministère ${ }^{39}$. Les principales sont la Loi sur la qualité de l'environnement (chap. Q-2), la Loi sur la conservation du patrimoine naturel (chap. C-61.01), la Loi sur le développement durable (chap. D-8.1.1) et la Loi sur les espèces menacées ou vulnérables (chap. E-12.01). Les règlements sont 
en lien avec l'évaluation et l'examen des impacts sur l'environnement de certains projets; les exploitations agricoles; les fabriques de pâtes et papiers; la gestion des déchets biomédicaux, déchets solides et matières dangereuses, etc. À la suite d'une plainte à caractère environnemental, un inspecteur peut faire une vérification sur le terrain à des fins de contrôle. La Loi sur la qualité de l'environnement encadre les recours possibles par le Ministère envers les personnes ou entreprises fautives.

\section{Conclusion}

La littérature en matière de divulgation et de protection des lanceurs d'alerte évolue rapidement, mais demeure tributaire de sa jeunesse relative. Puisque le discours universitaire se trouve forcément en mode rattrapage par rapport à la variété des études scientifiques, des doctrines juridiques ainsi que des pratiques sociales et législatives dans le monde, on ne saurait en proposer une synthèse exhaustive.

Cet article, au contenu plus normatif, avait pour objet de distinguer les types de comportements d'alerte, leur légitimité et leur utilité tant sur les plans social qu'organisationnel. En matière d'alerte, on doit retenir que les législateurs fédéral et québécois ont enrichi la protection des lanceurs d'alerte à plusieurs égards au fil des ans. Par contre, cette protection pourrait encore être améliorée, car elle présente certaines lacunes, et l'effectivité du droit dans ce domaine est souvent mise à mal.

Tout d'abord, la définition même de lanceur d'alerte (ou divulgateur ou dénonciateur) est floue, incertaine et diffère d'une législation à une autre (MartinBariteau et Newman, 2018). Le même problème se pose concernant les nombreuses définitions proposées par les législateurs des actes répréhensibles qui peuvent faire l'objet d'une divulgation. Ainsi, il s'avère très risqué pour une personne de faire une alerte dans un tel contexte, à moins qu'elle ait des connaissances solides en droit. L'appréciation de sa démarche devient délicate et anxiogène. Le lanceur d'alerte peut à tout moment se voir reprocher une faute dans le cadre de son travail ou être poursuivi pour diffamation (Leclerc, 2015).

Or, la législation fédérale actuelle vise essentiellement à protéger les employés du secteur public. Ceux du secteur privé ne sont protégés que par des dispositions fragmentées dans certaines lois ou certains programmes (Vatanchi, 2019). Quelques États fédérés canadiens (provinces), soit la Saskatchewan et le Nouveau-Brunswick, ont adopté des protections législatives pour les lanceurs d'alerte, mais ce n'est malheureusement pas le cas au Québec. Pourtant, selon Cordis et Lambert (2017), lorsque des pays ou des États adoptent des lois sur les lanceurs d'alerte qui couvrent les employés du secteur privé, ces dernières ont un effet dissuasif sur la fraude en entreprise.

Par ailleurs, même si les lanceurs d'alerte employés du secteur public sont mieux protégés que ceux du secteur privé par les différentes lois fédérales et québécoises, l'efficacité des processus de plainte laisse souvent à désirer ainsi que le rôle et l'encadrement des responsables du suivi des divulgations. La protection de la confidentialité des lanceurs d'alerte, l'offre d'accompagnement et le soutien requis, notamment sur le plan financier, font aussi défaut. Plusieurs observateurs demandent que la «bonne foi » exigée d'un lanceur d'alerte lors d'une divulgation soit abrogée et remplacée par une condition moins lourde, soit la « croyance raisonnable » du caractère répréhensible d'un acte (Comité permanent des opérations gouvernementales et des prévisions budgétaires, 2017; Martin-Bariteau et Newman, 2018). De plus, d'autres lois qui protègent la confidentialité, les secrets commerciaux, le devoir de loyauté du salarié ou encore le devoir de réserve viennent souvent limiter, sinon rendre incertaines les protections du lanceur d'alerte. La situation chaotique qu'a vécue l'agronome Louis Robert dans le traitement de son dossier en est un exemple éloquent.

Par contre, il faut être conscients que, même si elles sont protégées par des lois plus efficientes et plus faciles à appliquer, il faudra toujours une bonne dose de courage aux personnes qui décideront de sortir de l'anonymat et de prendre la parole pour défendre l'intérêt public au nom de la vérité. $\mathrm{La}$ solitude ainsi que l'isolement inévitable de ceux qui osent dénoncer les scandales, les injustices ou les dysfonctionnements peuvent devenir très lourds et s'apparenter souvent à un suicide social, personnel et financier. Pourtant, les programmes de divulgation dans les organisations publiques et privées constituent des outils de gestion non négligeables dans la lutte contre la corruption, la protection du public et de l'environnement. Sur le plan collectif, 
nous avons le devoir de ne pas les laisser seuls face à ce fardeau et de leur procurer le soutien psychologique et financier nécessaire.

Dans leur rapport personnel publié en novembre 2016 sur les paradis fiscaux et sur le monde de l'offshore, le prix Nobel d'économie Joseph Stiglitz et l'expert en anticorruption Mark Pieth formulent une douzaine de recommandations, dont celle de protéger davantage les lanceurs d'alerte ${ }^{40}$. Ils considèrent que «tous les pays devraient protéger les lanceurs d'alerte parmi les employés des secteurs publics et privés réglementés» (Stiglitz et Pieth, 2016, p. 19 trad. libre). Le besoin d'ériger un monde vivable rend nécessaire d'accorder le soutien voulu à l'expression critique, porteuse de visions de développement à l'échelle de la planète, et ce, peu importe le statut des personnes, employées ou pas, des secteurs public ou privé.

\section{REMERCIEMENTS}

Nous voulons, avec cet article, rendre hommage au sociologue Marc-André Morency. Il avait commencé cette réflexion sur les lanceurs d'alerte avec l'auteure principale quelques mois avant son décès en mars 2019. Spécialiste en sociologie du développement, Marc-André a été professeur-chercheur, directeur du Département des sciences humaines de l'UQAC, responsable du Groupe de recherche et d'interventions régionales (GRIR) et un membre actif et enthousiaste de la revue Organisations \& Territoires. À travers tous ces rôles, il n'a cessé de démontrer son attachement à l'Université du Québec à Chicoutimi, puis de stimuler les étudiantes et étudiants dans leurs apprentissages et la réalisation de leurs rêves.

\section{NOTES}

1 «Si vous constatez quelque chose, dites-le» ne devrait pas être seulement à l’avantage des forces de police, mais devrait s'appliquer dans tous les cas marqués par le secret lorsque l'intérêt public est en jeu» [trad. libre].

2 «L'image évoquée par whistle blower est celle des policiers des villes anglaises qui se promènent à pied dans les quartiers urbains. Lors d'un événement requérant la mobilisation d'autres forces de police, ils alertent leurs collègues à l'aide de leur sifflet» (Cailleba, 2019, p. 236).

3 «Selon un rapport de 2016 publié par l'Association of Certified Fraud Examiners (ACFE), qui a analysé au moins 2410 cas de fraude professionnelle dans plus de 114 pays, 39,1 \% des cas de fraude sont détectés par un lanceur d'alerte. Le rapport indique que les employés sont à l'origine de près de la moitié de tous les cas de divulgation qui conduisent à la détection de fraude, suivis des clients (17,8\%), des anonymes (14\%), des vendeurs $(9,9 \%)$, des actionnaires/propriétaires $(2,7 \%)$ et des concurrents $(1,6 \%)$. La divulgation apparait donc comme un moyen efficace de braquer les projecteurs sur la fraude professionnelle» (Smaili et Arroyo, 2019, p. 95, trad. libre).

4 Devant l'absence d'uniformité dans l'usage des termes pour désigner ce phénomène de prise de parole, nous utiliserons la notion de «divulgation». Pour désigner la personne qui divulgue des actes répréhensibles, nous utiliserons l'expression «lanceur d'alerte», même si cette expression n’est pas encore consacrée dans le corpus législatif canadien et québécois. Nous la trouvons plus parlante pour les non-initiés que les termes « divulgateur» ou «dénonciateur». De plus, la commission Charbonneau (Charbonneau et Lachance, 2015) en a fait usage près de 60 fois dans la rédaction de son rapport, et cette expression est désormais popularisée par les journalistes, les militants et plusieurs instances internationales. Cependant, lorsque nous analyserons une loi particulière tant au fédéral qu'au Québec, nous allons respecter la terminologie précise utilisée par le législateur.

5 À titre d'exemple, les membres du Whistleblowing International Network (WIN) fournissent des conseils, des outils et de l'expertise dont ont besoin les personnes travaillant dans différents pays pour lutter contre la corruption, le gaspillage, la fraude, les abus, l'illégalité et les menaces à l'endroit du bien public. (Source : https://whistleblowingnetwork.org)

6 Notre analyse ne visera pas les situations de dénonciation (ou de signalement) entre professionnels d'un même ordre ni les signalements faits en vertu de la Loi sur la protection de la jeunesse (LRQ, chap. P-34.1) ou de la Loi visant à lutter contre la maltraitance envers les aînés et toute autre personne majeure en situation de vulnérabilité (LLRQ, chap. L-6.3).

7 Un bel exemple de dénonciateur interne est celui de l'agronome Louis Robert, fonctionnaire au MAPAQ, qui a dénoncé la mainmise de l'industrie des pesticides sur le Centre de recherche sur les grains, un organisme largement subventionné par l'État.

8 Pour différencier les dénonciateurs internes des dénonciateurs externes, certains désignent ces derniers comme des sonneurs d'alarme (bell-ringers) (Miceli et collab., 2014).

9 À titre d'exemple, les sous-traitants peuvent désormais avoir un accès privilégié à des renseignements confidentiels ou à des faits répréhensibles commis chez leurs clients qui, auparavant, n'étaient connus que des membres de l'organisation concernée (Culiberg et Mihelic, 2017). Le même constat s'applique aux clients ou aux consommateurs, qui sont souvent touchés par des actes répréhensibles, dont le non-respect des promotions, les publicités mensongères, les prix gonflés, la technique des prix d'appel, la vente sous pression ou encore les clauses et les frais cachés (Culiberg et Mihelic, 2017). 
10 «En vaquant à vos occupations chez votre employeur, vous apprenez l'imminence d'un vol dans l'entrepôt. Ou encore : vous entendez l'un de vos collègues se vanter d'avoir pu encore une fois, sous de faux prétextes, obtenir le remboursement d'un compte de dépenses erroné ou frauduleux » (Bonhomme et Paquette, 2006, p. 169).

11 Les acteurs de la démocratie monitoire estiment que «veiller, être en état d'alerte, être sur ses gardes sont des attributs essentiels de la citoyenneté. Des attributs originaires aussi : le citoyen antique ne pouvait s'imaginer sous les seules espèces de l'électeur épisodique» (Benyekhlef, 2017, p. 20, citant Rosanvallon, 2006, p. 39).

12 Au Canada, l'encadrement légal des médias et des journalistes comprend la Charte québécoise des droits et libertés de la personne, la Charte canadienne des droits et libertés ainsi que la Loi sur la protection des sources journalistiques. Cette dernière a modifié, en octobre 2017, la Loi sur la preuve au Canada ainsi que le Code criminel en reconnaissant au journaliste le droit de s'opposer à la divulgation d'un renseignement ou d'un document qui permettrait de dévoiler l'identité d'une source confidentielle. Il incombe désormais à la personne qui demande la divulgation d'un tel renseignement ou document d'établir qu'elle ne peut pas être mise en preuve par un autre moyen raisonnable et que l'intérêt public dans l'administration de la justice l'emporte sur l'intérêt public de préserver la confidentialité de la source journalistique (paragr. 39(1) et 39(2) de la Loi sur la preuve au Canada). Cette loi a récemment fait l'objet d'un jugement de la Cour suprême du Canada dans le cas de la journaliste d'enquête Marie-Maude Denis de l'émission Enquête de Radio-Canada (Denis c. Côté, [2019] CSC 44). La Cour suprême a infirmé une décision de la Cour du Québec qui contraignait la journaliste de divulguer le nom de la personne qui lui avait transmis de l'information compromettante à l'égard de Marc-Yvan Côté, accusé au criminel. Monsieur Côté alléguait qu'il n'avait pas un procès juste et équitable s'il n'avait pas le nom de cette personne.

13 Le cas célèbre de la Canadienne Karla Homolka, épouse et complice du tueur en série Paul Bernardo, a soulevé la grogne populaire au milieu des années 1990. Afin d'obtenir une peine réduite, elle a pactisé avec les autorités, avec pour résultat de faire condamner Bernado à la prison à vie.

14 Voir par exemple Merk c. Association internationale des travailleurs en ponts, en fer structural, ornemental et d'armature, section locale 771, [2005] CSC 70; Haydon c. Canada (1re inst.), [2001] 2 CF 82; Anderson c. IMTT-Québec inc., [2013] CAF 90; Rochon c. Tribunal administratif du travail, [2017] QCCS 5202; Syndicat de la fonction publique et parapublique du Québec (SFPQ) c. Gouvernement du Québec (ministère du Développement durable, de l'Environnement et de la Lutte contre les changements climatiques), [2017] QCTA 61.

15 Par exemple, au Québec, en matière de liberté académique, la Fédération québécoise des professeures et professeurs d'université recommande d'appliquer aux personnes professeures une forme de dérogation au devoir de loyauté prévu notamment à l'article 2088 du Code civil du Québec. FQPPU (2020, 23 octobre). Consultation publique sur l'université québécoise du futur (positions et propositions de la FQPPU). https:// fqppu.org/wp-content/uploads/2020/10/UFutur_consultation_FQPPU_final.pdf

16 False Claim Act de 1863; National Labor Relations Act de 1935; Whistleblower's Protection Act de 1994; Sarbanes-Oxley Act de 2002; Dodd-Frank Wall Street Reform and Consumer Protection Act de 2010.

17 Public Interest Disclosure Act de 1998.

18 «[...] À la connaissance de David Hutton, chargé de recherche à la Whistleblowing Initiative du Centre for Freedom of Expression de l'Université Ryerson, la disposition [425.1 du Code criminel] n'a jamais été appliquée. “Celui qui sonne l'alarme n'a aucun recours contre ceux qui tirent les ficelles; c'est à la police d'intervenir. Mais, par définition, le divulgateur révèle un secret que le pouvoir en place ne veut pas ébruiter; alors, difficile de croire que les forces de l'ordre voleront à son secours." » (Toub, 2020, s. p.). Dans l'affaire Anderson c. IMTT-Québec inc. ([2013] CAF 90), «la Cour d'appel fédérale a refusé la protection à un employé qui a déposé une plainte hâtive et vexatoire sans avoir d'abord vérifié les mesures prises ou attendu l'issue des mécanismes internes » (Martin-Bariteau et Newman, 2018, p. 17).

19 Voir le Rapport du Groupe de travail sur la divulgation des actes fautifs de 2004 de l'Agence de gestion des ressources humaines de la fonction publique du Canada ainsi que le rapport factuel de 2005 Qui est responsable? de la Commission d'enquête sur le programme de commandites et les activités publicitaires, menée par le juge John H. Gomery, commissaire.

20 La LPFDAR ne protège pas ceux qui travaillent dans les Forces armées canadiennes, à la sécurité des communications, ni au Service canadien du renseignement de sécurité. Cependant, ces groupes sont tenus de créer des mécanismes de divulgation interne en vertu de l'article 52 de la LPFDAR.

21 La LPFDAR prévoit que le Conseil du Trésor doit définir un code de conduite applicable à la fonction publique dans son ensemble (art. 5). Le Code de valeurs et d'éthique du secteur public, entré en vigueur le 2 avril 2012, précise les valeurs (respect de la démocratie, respect des personnes, intégrité, intendance, excellence) et les comportements devant être adoptés par les fonctionnaires dans l'exercice de leurs fonctions professionnelles (SCT, 2011).

22 Detorakis c. Canada (Procureur général), [2010] CF 39; Agnaou [no 1] c. Canada (Procureur général), [2014] CF 86.

23 À tout moment au cours de l'enquête, l'enquêteur peut recommander au commissaire de nommer un conciliateur chargé de tenter d'en arriver à un règlement de la plainte (art. 20). Si un règlement fait l'objet d'une entente et qu'il se trouve approuvé par le commissaire (art. 20.2), la plainte du fonctionnaire est alors rejetée ou retirée.

24 Agnaou [no 2] c. Canada (Procureur général), [2014] CF 87. 
25 Bron (2019) remarque qu'il est difficile de juger de l'efficacité des régimes de divulgation avec comme seules preuves disponibles l'information provenant de ces rapports annuels. Ceux-ci sont rédigés bien souvent par les personnes chargées de la mise en œuvre des régimes, fournissent un strict minimum de détails sur les plaintes et ne donnent pas le contexte de la divulgation.

26 Décret 114-2011 du 16 février 2011. L’UPAC n'est pas un corps policier. Parmi ses partenaires, on retrouve : la Commission de la construction du Québec, le ministère des Affaires municipales, des Régions et de l'Occupation du territoire, la Régie du bâtiment du Québec, l'Unité anticollusion du MTQ, l'escouade Marteau et l'Agence du revenu du Québec. Pour une description complète du mandat de l'UPAC, consulter le site du Commissaire à la lutte contre la corruption : www.upac.gouv.qc.ca/upac/mandat.html

27 Elle s'applique aux organismes publics, aux établissements d'enseignement de divers ordres, aux centres de services scolaires, aux centres de la petite enfance, aux établissements publics ou privés visés par la Loi sur les services de santé et les services sociaux (RLRQ, chap. S-4.2) et aux municipalités.

28 En septembre 2012, l'Assemblée nationale a adopté la Loi sur l'intégrité en matière de contrats publics (RLRQ, chap 25), qui a apporté d'importantes modifications à la Loi sur les contrats des organismes publics (RLRQ, chap. C-65.1). Le cœur de cette loi prévoit une nouvelle procédure administrative par laquelle les entreprises qui souhaitent soumissionner sur des marchés publics doivent obtenir de l'Autorité des marchés financiers (AMF) un certificat d'intégrité. Sans ce certificat, l'entreprise ne peut pas soumissionner. La décision de l'AMF d'accorder le certificat repose sur un mélange de facteurs objectifs et subjectifs (SCT, 2014).

29 L'amende est de 2000 \$à 20000 \$ s'il s'agit d'une personne physique; de 10000 \$à 250000 \$ s'il s'agit d'une personne morale. En cas de récidive, ces amendes sont portées au double (art. 33).

30 La Loi sur les normes du travail entend par «salarié » une personne qui travaille pour un employeur et qui a droit à un salaire. Le travailleur autonome n'est donc pas visé par cette loi.

31 La Loi sur les normes du travail prévoit spécifiquement qu’il est interdit à un employeur ou à son agent de congédier, de suspendre ou de déplacer un salarié, d'exercer à son endroit des mesures discriminatoires ou des représailles ou de lui imposer toute autre sanction en raison d'une divulgation d'un acte répréhensible au sens de la Loi concernant la lutte contre la corruption ou de la collaboration à une vérification ou enquête portant sur un tel acte [paragr. 122(7)].

32 D 1029-2011, GOQ 2011.II.4767.

33 Pour leur part, les lanceurs d'alerte municipaux verront toutefois leur plainte adressée au Protecteur du citoyen référée au ministre responsable des Affaires municipales, si notamment un acte répréhensible a été commis ou est sur le point de l'être dans le cadre d'un processus d'adjudication ou d'attribution d'un contrat d'un organisme public ou dans le cadre de l'exécution d'un tel contrat, incluant l'octroi d'une aide financière [art. 17.1].

Ainsi, si cette divulgation concerne une contravention à une loi ou à un règlement à l'égard d'un processus d'adjudication, d'attribution ou d'exécution d'un contrat public visé au paragraphe 20(1) de la Loi sur l'Autorité des marchés publics (RLRQ, chap. A-33.2.1), le Protecteur du citoyen doit se dessaisir du traitement de la plainte à ce sujet, tout en ayant l'obligation de transmettre les informations ainsi reçues à l’autorité responsable [art. 14].

Par ailleurs, les lanceurs d'alerte municipaux peuvent s'adresser directement à l'Autorité des marchés publics (art. 56 de la Loi sur l'Autorité des marchés publics. Ils jouiront alors des protections contre les représailles de leur employeur (art. 58 et 61-65 de la Loi sur l'Autorité des marchés publics et paragr. 122(11) de la Loi sur les normes du travail.

Enfin, si la divulgation concerne un manquement en matière d'éthique et de déontologie applicable à un membre d'un conseil d'une municipalité, les lanceurs d'alerte municipaux devront s'adresser, cette fois, à la Commission municipale du Québec (art. 20 et suivants de la Loi sur l'éthique et la déontologie en matière municipale, RLRQ, chap. E-15.1.0.1).

34 La LNT [paragr. 122(11)] prévoit la possibilité de déposer une plainte pour un divulgateur salarié contre son employeur devant la CNESST.

35 En avril 2021, Louis Robert a publié un livre où il dénonce l'ingérence des compagnies de pesticides et d'engrais dans la recherche publique (Robert, 2021).

36 En réponse à différents scandales financiers (Enron, Tyco, Adelphia, WorldCom), la Sarbanes-Oxley Act (SOX), adoptée en 2002 par le Congrès américain, «impose notamment aux sociétés américaines (et à leurs filiales à l'étranger) ainsi qu'aux sociétés étrangères cotées à la Bourse de New York de mettre en place un code d’éthique et un système permettant aux salariés de rapporter anonymement les fraudes et les malversations comptables et financières dont ils ont connaissance» (De Bry, 2008, p. 143). Cette loi, qui vise l'amélioration de la gouvernance de ces sociétés, prévoit des peines de prison envers toute personne qui prendrait des mesures pour empêcher un salarié de dénoncer une telle situation aux autorités compétentes (art. 301, 404, 806 et 1107).

37 Voir la description du programme de l'AMF à l'adresse suivante : https://lautorite.qc.ca/grand-public/assistance-et-plainte/programme-de-denonciation-lanceurs-dalerte 
38 Parmi ces lois, on retrouve les suivantes : Loi sur les assureurs (chap. A-32.1), Loi sur les coopératives de services financiers (chap. C 67.3), Loi sur la distribution de produits et services financiers (chap. D-9.2), Loi sur les institutions de dépôts et la protection des dépôts (chap. I-13.2.2), Loi sur les instruments dérivés (chap. I-14.01), Loi sur les sociétés de fiducie et les sociétés d'épargne (chap. S 29.02), Loi sur les valeurs mobilières (chap. V-1.1), Loi sur le mouvement Desjardins (2000, chap. 77) et Loi sur l'assurance automobile (Titre VII, chap. A-25).

39 Il peut s'agir, par exemple, d'un épandage de lisier trop près d'un puits d'eau potable, de matières résiduelles dangereuses mal entreposées, de fumée noire provenant de la cheminée d’une usine ou encore de la présence de machinerie lourde dans le lit d’un cours d'eau.

40 «Le prix Nobel d'économie Joseph Stiglitz et l'expert anticorruption suisse Mark Pieth avaient quitté avec fracas, en août [2016], le comité de spécialistes mis sur pied par le Panama après les révélations des Panama Papers d'avril, en dénonçant des velléités de censure du président du pays, Juan Carlos Varela. Ils publient [...] le fruit de leurs réflexions poursuivies en solo sur les paradis fiscaux et le monde de l'offshore dans un rapport intitulé Vaincre l'économie de l'ombre » (Michel, 2016, s. p.; voir Stiglitz et Pieth, 2016).

\section{RÉFÉRENCES}

Andrade, J. A. (2015). Reconceptualising whistleblowing in a complex world. Journal of Business Ethics, 128, 321-335. www.jstor.org/ stable/24702873

Autorité des marchés financiers (AMF). (2017). Rapport annuel 2016-2017. AMF. http://www.assnat.qc.ca/Media/ Process.aspx?MediaId=ANQ.Vigie.Bll.DocumentGenerique_132283\&process=Original\&token= ZyMoxNwUn8ikQ+TRKYwPCjWrKwg+vIv9rjij7p3xLGTZDmLVSmJLoqe/vG7/YWzz

Autorité des marchés financiers (AMF). (2020). Rapport anmuel 2019-2020. AMF. https://lautorite.qc.ca/fileadmin/lautorite/ grand_public/publications/organisation/rapports-annuels/autorite/amf-rapport-annuel-2019-2020.pdf

Ayers, S. et Kaplan, S. E. (2005). Wrongdoing by consultants: An examination of employees' reporting intentions. Journal of Business Ethics, 57(2), 121-137. https://doi.org/10.1007/S10551-004-4600-0

Bégin, L., Brodeur, P.-O., Lalonde, P., Ouimet, G., St-Martin, D., Trent, P. et Valois, M. (2016). Rapport du Comité de suivi public des recommandations de la commission Charbonneau. Éthique publique, 18(2). https://doi.org/10.4000/ethiquepublique.2789

Benyekhlef, K. (2017). Au-delà de la représentation : les figures de la démocratie. Thémis.

Biron, J. et Rousseau, S. (2012). «Whistleblowing», divulgation, dénonciation... Vers une meilleure surveillance des sociétés. Revue du Barreau canadien, 91, 657-699. https://cbr.cba.org/index.php/cbr/article/download/4307/4300

Boisvert, A.-M. (2005). La protection des collaborateurs de la justice : éléments de mise à jour de la politique québécoise : rapport final présenté au ministre de la Sécurité publique. Gouvernement du Québec. www.securitepublique.gouv.qc.ca/fileadmin/Documents/police/ publications/boisvert/rapport_boisvert_2005.pdf

Bonhomme, R. et Paquette, S.-P. (2006). Existe-t-il vraiment un conflit entre l'obligation de loyauté et l'obligation de divulgation interne et externe en milieu de travail? Dans Barreau du Québec (dir.), Développements récents en droit du travail (vol. 245, p. 167-221). Éditions Yvon Blais. https://edoctrine.caij.qc.ca/developpements-recents/245/367144711/\#infos

Bowal, P. (2011). Retaliation against whistleblowers is a crime. Lawnow, 36(2), 28-34. http://hdl.handle.net/1880/49691

Bron, I. (2019). Vile wretches and public heroes: A survey of Canadian whistleblowing literature. Canadian Public Administration, 62(2), 356-361. https://doi.org/10.1111/capa.12324

Brown, A. J. (2013). Towards "ideal” whistleblowing legislation? Some lessons from recent Australian experience. E-Journal of International and Comparative Labour Studies, 2(3). http://ejcls.adapt.it/index.php/ejcls_adapt/article/view/134

Brunelle, C. et Samson, M. (2005). La liberté d'expression au travail et l'obligation de loyauté du salarié : plaidoyer pour un espace critique accru. Les Cabiers de droit, 44(4), 795-990. https://doi.org/10.7202/043869ar

Cailleba, P. (2019). Quel risque pour le lanceur d'alerte? L'accusation de déloyauté. Recherche en sciences de gestion, 132(3), 235-257. https://doi.org/10.3917/resg.132.0235

Charbonneau, F. et Lachance, R. (2015). Rapport final de la Commission d'enquête sur l'octroi et la gestion des contrats publics dans lindustrie de la construction (4 tomes). Gouvernement du Québec. www.ceic.gouv.qc.ca/fileadmin/Fichiers_client/fichiers/Rapport_final/

Rapport_final_CEIC_Integral_c.pdf 
Chateauraynaud, F. (2013). Lanceur d'alerte. Dans I. Casillo, R. Barbier, L. Blondiaux, F. Chateauraynaud, J.-M. Fourniau, R. Lefebvre, C. Neveu et D. Salles (dir.), Dictionnaire critique et interdisciplinaire de la participation. GIS Démocratie et Participation. https://www.dicopart.fr/fr/dico/lanceur-dalerte

Clark, K. (2012). White paper on the law of whistleblowing. https://dx.doi.org/10.2139/ssrn.2176293

Comité permanent des opérations gouvernementales et des prévisions budgétaires. (2017). Renforcer la protection de l'intérêt public dans la Loi sur la protection des fonctionnaires divulgateurs d'actes répréhensibles : rapport du Comité permanent des opérations gouvernementales et des prévisions budgétaires. Chambre des communes du Canada. www.noscommunes.ca/Content/Committee/421/OGGO/Reports/ RP9055222/oggorp09/oggorp09-f.pdf

Comte-Sponville, A. (2017, 23 octobre). «Balance ton porc» : la délation n’est pas la dénonciation. Challenges. www.challenges.fr/ politique/balance-ton-porc-lance-a-la-suite-de-l-affaire-weinstein-jette-en-pature-des-dizaine-de-noms_508305

Cordis, A. et Lambert, E. (2017). Whistleblower laws and corporate fraud: Evidence from the United States. Accounting Forum, 41(4), 289-299. https://doi.org/10.1016/j.accfor.2017.10.003

Culiberg, B. et Mihelic, K. K. (2017). The evolution of whistleblowing studies: A critical review and research agenda. Journal of Business Ethics, 146, 787-803. https://doi.org/10.1007/s10551-016-3237-0

De Bry, F. (2008). Salariés courageux oui, mais héros ou délateurs? Du whistleblowing à l'alerte éthique. Revne internationale de psychosociologie, XIV(34), 135-150. https://doi.org/10.3917/rips.034.0135

De Maria, W. (1995). Whistleblowing. Alternative Law Journal, 20(6), 270-281. http://classic.austlii.edu.au/au/journals/AltLawJl/ 1995/117.html

De Quenaudon, R. (2015). Chapitre 15. Les lanceurs d'alerte. Dans A. Supiot (dir.), Prendre la responsabilité au sérieux (p. 291-307). Presses Universitaires de France. https://doi.org/10.3917/puf.delma.2015.03.0291"

Desjardins, J.-P. et Jutras, M. (2007). L'impact du scandale des commandites sur la régulation des comportements des agents publics du gouvernement canadien. ENAP. http://archives.enap.ca/bibliotheques/2008/02/030017511.pdf

Dugas, R. (2013). Divulgation d'actes répréhensibles et protection contre les représailles : les expériences fédérale et québécoise. Dans Barreau du Québec (dir.), Développements récents en droit du travail (vol. 364, p. 67-101). Éditions Yvon Blais. https://edoctrine.caij.qc.ca/developpements-recents/364/368126848

Dyck, A., Morse, A. et Zingales, L. (2007). Who blows the whistle on corporate fraud? The Journal of Finance, 65(6), 2213-2253. https://www.nber.org/system/files/working_papers/w12882/w12882.pdf

Ferguson, G. (2018). Global corruption: Law, theory \& practice (3e éd.). University of Victoria. https://icclr.org/wp-content/ uploads/2019/06/2018CanLIIDocs28-1.pdf?x68316

Fines, L. (2013). Les crimes invisibles : délits contemporains, dénonciations et temps de réaction. Édition Liber.

Foegle, J. (2016). Lanceur d'alerte ou «leaker»? Réflexions critiques sur les enjeux d’une distinction. Revne des droits de l'homme, 10, 1-17. https://doi.org/10.4000/revdh.2367

Groupe de travail sur l'administration de la justice en matière criminelle. (1991). Rapport (rapport Guérin), Gouvernement du Québec, ministère de la Justice et ministère de la Sécurité publique.

Hartmann, F. (2014). Lanceurs d'alerte : les mauvaises consciences de nos démocraties. Don Quichotte.

Jubb, P. B. (1999). Whistleblowing: A restrictive definition and interpretation. Journal of Business Ethics, 21, 77-94. https://doi.org/10.1023/A:1005922701763

Karim, V. (2020). Les obligations (vol. 1, 5e éd.). Wilson \& Lafleur.

Keith, N. (2017). Canadian anti-corruption law and compliance. LexisNexis.

Lachapelle, A. (2019). La protection des lanceurs d'alerte (whistleblowers) à l'heure d'Internet. Dans Q. Van Enis et C. De Terwangne (dir.), L'Europe des droits de l'Homme à l'heure d'Internet (p. 223-269). Bruylant. http://www.crid.be/pdf/public/8438.pdf

Laframboise, H. L. (1991). Vile wretches and public heroes: The ethics of whistleblowing in government. Canadian Public Administration, 34(1), 73-77. https://doi.org/10.1111/j.1754-7121.1991.tb01438.x 
Lavite, C. (2016). L'approche socio-légale de la protection des lanceurs d'alerte : pistes de réflexion pour un enrichissement sociologique de l'analyse juridique. Revue des droits de l'homme, 10, 1-15. https://doi.org/10.4000/revdh.2648

Leclerc, O. (2015). Lanceur d'alerte. Dans Emmanuel Henry (dir.), Dictionnaire critique de l'expertise : santé, travail, environnement (p. 194-202). Presses de Sciences Po. https://doi.org/10.3917/scpo.henry.2015.01.0194

Lévesque, L. (2014, 29 octobre). Les lanceurs d’alerte négligés au Canada. Le Devoir. https://www.ledevoir.com/societe/422381/ commission-charbonneau-les-lanceurs-d-alerte-negliges-au-canada

Lewis, D. (2011). Whistleblowing in a changing legal climate: Is it time to revisit our approach to trust and loyalty at the workplace? Business Ethics: A European Review, 20(1), 71-87. https://doi.org/10.1111/j.1467-8608.2010.01609.x

Lochak, D. (2016). Les lanceurs d'alerte et les droits de l'Homme : réflexions conclusives. Revne des droits de l'homme, 10. https://doi.org/10.4000/revdh.2362

Martin-Bariteau, F. et Newman, V. (2018). Dénonciation au Canada : une synthèse des connaissances [document de travail no 2018-07]. Université d'Ottawa, Faculté de droit d'Ottawa.

Mayr, C. et Struthers, S. J. (2017). Projet de loi 141 : les amendements proposés à la Loi sur l'Autorité des marchés financiers relatifs aux dénonciations. https://www.mccarthy.ca/fr/references/articles/projet-de-loi-141-les-amendements-proposes-la-loi-sur-lautorite-des-marchesfinanciers-relatifs-aux-denonciations

Miceli, M. P., Dreyfus, S. et Near, J. P. (2014). Outsider "whistleblowers": Conceptualizing and distinguishing "bell-ringing" behavior. Dans A. J. Brown, D. Lewis, R. Moberly et W. Vandekerchhove (dir.), International Handbook on whistleblowing research (p. 71-94). Edward Elgar.

Michel, A. (2016, 15 novembre). Les recommandations de Joseph Stiglitz et Mark Pieth pour lutter contre les paradis fiscaux. Le Monde. www.lemonde.fr/economie/article/2016/11/15/les-recommandations-de-joseph-stiglitz-et-mark-pieth-pour-luttercontre-les-paradis-fiscaux_5031592_3234.html

Near, J. P. et Miceli, M. P. (1995). Effective whistle-blowing. Academy of Management Review, 20(3), 679-708. https://doi.org/10.2307/258791

O’Bomsawin, F. et Marcoux, L. (2014). La divulgation d'actes répréhensibles. Dans Conférence des arbitres du Québec (dir.). Les cabiers de la Conférence des arbitres du Québec: débats d'actualité (p. 79-108). Wilson \& Lafleur.

Piraux, A. (2008). L'éthique administrative à l'épreuve de ses usages. Pyramides, 16(1), 83-144. http://journals.openedition.org/ pyramides $/ 197$

Protecteur du citoyen (2019a). Application de la Loi facilitant la divulgation d'actes réprébensibles à l'égard des organismes publics: des manquements majeurs de la part du ministère de l'Agriculture, des Pếcheries et de l'Alimentation [rapport spécial du Protecteur du citoyen]. Assemblée nationale. https://protecteurducitoyen.qc.ca/sites/default/files/pdf/rapports_speciaux/rapport-mapaq-manquementstraitement-divulgation.pdf

Protecteur du citoyen (2019b). Bilan du Protecteur du citoyen sur la mise en ceuvre de la Loi facilitant la divulgation d'actes réprébensibles à l'égard des organismes publics. Assemblée nationale. https://protecteurducitoyen.qc.ca/sites/default/files/pdf/rapport-bilan-loi-divulgationactes-reprehensibles.pdf

Rancourt, G. et Cloutier, V. (2008). L'obligation de loyauté et de dénonciation publique de l'employeur. Ordre des conseillers en ressources humaines agréés. https://ordrecrha.org/ressources/TBD/Archives/Vigie-RT/l-obligation-de-loyaute-et-la-denonciationpublique-de-l-employeur

Robert, L. (2021). Pour le bien de la terre. Éditions MultiMondes.

Robitaille-Rodriguez, J. (2017). Les récompenses financières favorisent-elles la dénonciation? Le Québec et l'Ontario : deux approches à l'essai. Stikeman Elliott. https://www.stikeman.com/fr-ca/savoir/droit-canadien-valeurs-mobilieres/Les-recompenses-financieres-favorisent-ellesla-denonciation

Rosanvallon, P. (2006). La contre-démocratie : la politique à l'âge de la défiance. Seuil.

Russell, M.-A. (2019). Entrée en fonction de l'Autorité des marchés publics : quel impact pour les municipalités. Dans Barreau du Québec (dir.), Développements récents en droit municipal (vol. 456, p. 67-102). Éditions Yvon Blais. https://edoctrine.caij.qc.ca/ developpements-recents/456/369022500/ 
Sasseville, A. et Samoisette Fournier, G. (2017). L'employé sonneur d'alarme dans la lutte contre la corruption : outil efficace ou obstacle à la gestion des ressources humaines. Dans Barreau du Québec (dir.), Développements récents en droit du travail (vol. 429, p. 4171). Éditions Yvon Blais. https://edoctrine.caij.qc.ca/developpements-recents/429/368936010

Schehr, S. (2008). L'alerte comme forme de déviance : les lanceurs d'alerte entre dénonciation et trahison. Déviance et société, 32(2), 149-162. https://doi.org/10.3917/ds.322.0149

Secrétariat du Conseil du Trésor (SCT) du Canada. (2011). Code de valeurs et d'éthique du secteur public. Gouvernement du Canada. https://www.tbs-sct.gc.ca/pol/doc-fra.aspx?id=25049

Secrétariat du Conseil du Trésor (SCT) du Canada. (2014). Rapport concernant la mise en ceuvre de la Loi sur l'intégrité en matière de contrats publics. Gouvernement du Canada. https://www.tresor.gouv.qc.ca/fileadmin/PDF/faire_affaire_avec_etat/integrite/ rapport_integrite_contrats_publics.pdf

Secrétariat du Conseil du Trésor (SCT) du Canada. (2020). Rapport annuel concernant la Loi sur la protection des fonctionnaires divulgateurs d'actes répréhensibles de 2019 à 2020. Gouvernement du Canada. https://www.canada.ca/fr/secretariat-conseil-tresor/services/valeursethique/protection-divulgateurs/rapport-annuel-loi-protection-fonctionnaires-divulgateurs-actes-reprehensibles-2019-2020.html

Secrétariat du Conseil du trésor (SCT) du Québec. (2020). Loi facilitant la divulgation d'actes répréhensibles à l'égard des organismes publics: rapport sur la mise en cuure de la loi. Gouvernement du Québec. https:/ /www.tresor.gouv.qc.ca/fileadmin/PDF/publications/ Loi_divulgation_actes_reprehensibles.pdf

Shepherd, R. (2017). Whistle-blowing and ethical practice. Dans I. Greene et D. P. Shugarman (dir.), Honest Politics Now, James Lorimer \& Company Ltd.

Simard, J. et Morency, M.-A. (2013). La confiance vigilante : les instruments de la saine gouvernance. Organisations \& territoires, 21(3), 19-28. https://constellation.uqac.ca/3250

Smaili, N. et Arroyo, P. (2019). Categorization of whistleblowers using the whistleblowing triangle. Journal of Business Ethics, 157(1), 95-117. https://doi.org/10.1007/s10551-017-3663-7

Stiglitz, J. E. et Pieth, M. (2016). Overcoming the shadow economy. Friedrich-Ebert-Stiftung. http://library.fes.de/pdf-files/iez/12922.pdf

Tait, J. (1997). A strong foundation: Report of the task force on public service values and ethics. Canadian Public Administration, 40(1), 1-22. https://doi.org/10.1111/j.1754-7121.1997.tb01493.x

Tchotourian, I. (2016, 13 octobre). Droit, entreprise et citoyen, le blogue. Dénonciation : nouvel outil éthique des entreprises. http://www.contact.ulaval.ca/article_blogue/denonciation-nouvel-outil-ethique-des-entreprises/

Toub, M. (2020, 27 avril). Le Canada doit mieux protéger les lanceurs d'alerte. Magarine Pivot. Comptables professionnels agréés Canada. https://www.cpacanada.ca/fr/nouvelles/magazine-pivot/2020-04-27-canada-protection-denonciateurs

Trudel, P. (2019, 22 janvier). Loyauté déloyale. Le Devoir. https://www.ledevoir.com/opinion/chroniques/546010/loyaute-deloyale

Van Enis, Q. (2017). Une solide protection des sources journalistiques et des lanceurs d'alerte : une impérieuse nécessité à l'ère dite de la « post-vérité »? Dans Y. Ninane (dir.). Le secret (p. 95-152). Anthemis.

Vandekerkhove, W. et Commers, M. S. R. (2004). Whistleblowing and rational loyalty. Journal of Business Ethics, 53(1-2), 225-233. https://doi.org/10.1023/B:BUSI.0000039411.11986.6b

Vandekerkhove, W. et Tsahuridu, E. E. (2010). Risky rescues and the duty to blow the whistle. Journal of Business Ethics, 97, 365-380. https://doi.org/10.1007/s10551-010-0513-2

Vatanchi, S. (2019). Whistleblowing in Canada: A call for enhanced private sector protection. Western Journal of Legal Studies, 9(1), 1-15. https://doi.org/10.5206/uwojls.v9i1.6838

Wolfe, S., Worth, M. Dreyfus, S. et Brown, A.J. (2014). Whistleblower Protection Laws in G20 Countries: Priorities for Action. 